\title{
Simple factor dressing and the López-Ros deformation of minimal surfaces in Euclidean 3-space
}

\author{
K. Leschke ${ }^{1} \cdot$ K. Moriya ${ }^{2}$
}

Received: 11 January 2017 / Accepted: 16 April 2018 / Published online: 19 December 2018

(c) The Author(s) 2018

\begin{abstract}
The aim of this paper is to investigate a new link between integrable systems and minimal surface theory. The dressing operation uses the associated family of flat connections of a harmonic map to construct new harmonic maps. Since a minimal surface in 3-space is a Willmore surface, its conformal Gauss map is harmonic and a dressing on the conformal Gauss map can be defined. We study the induced transformation on minimal surfaces in the simplest case, the simple factor dressing, and show that the well-known López-Ros deformation of minimal surfaces is a special case of this transformation. We express the simple factor dressing and the López-Ros deformation explicitly in terms of the minimal surface and its conjugate surface. In particular, we can control periods and end behaviour of the simple factor dressing. This allows to construct new examples of doubly-periodic minimal surfaces arising as simple factor dressings of Scherk's first surface.
\end{abstract}

\section{Introduction}

Minimal surfaces, that is, surfaces with vanishing mean curvature, first implicitly appeared as solutions to the Euler-Lagrange equation of the area functional in [42] by Lagrange. The classical theory flourished through contributions of leading mathematicians including, amongst others, Catalan, Bonnet, Serre, Riemann, Weierstrass, Enneper, Schwarz and Plateau. By now, the class of minimal surfaces belongs to the best investigated and understood classes in surface theory. One of the reasons for the success of its theory is the link to Complex Anal-

K. Leschke was partially supported by DFG SPP 1154 “Global Differential Geometry” and JSPS KAKENHI Grant-in-Aids for Scientific Research (C), Grant number 24540090. K. Leschke and K. Moriya were supported by JSPS KAKENHI Grant-in-Aids for Scientific Research (C), Grant numbers 22540064 and 25400063.

$\triangle \quad$ K. Leschke

k.leschke@le.ac.uk

K. Moriya

moriya@math.tsukuba.ac.jp

1 Department of Mathematics, University of Leicester, University Road, Leicester LE1 7RH, UK

2 Division of Mathematics, Faculty of Pure and Applied Sciences, University of Tsukuba, 1-1-1 Tennodai, Tsukuba, Ibaraki 305-8571, Japan 
ysis: since a minimal conformal immersion $f: M \rightarrow \mathbb{R}^{3}$ from a Riemann surface $M$ into 3-space is a harmonic map, minimal surfaces are exactly the real parts holomorphic curves $\Phi: M \rightarrow \mathfrak{C}^{3}$ into complex 3-space. Due to the conformality of $f$, the holomorphic map $\Phi$ is a null curve with respect to the standard symmetric bilinear form on $\mathfrak{C}^{3}$. A particularly important aspect of this approach is that the Enneper-Weierstrass representation formula, see [27,73], allows to construct all holomorphic null curves, and thus all minimal surfaces, from the Weierstrass data $(g, \omega)$ where $g$ is a meromorphic function and $\omega$ a holomorphic 1 -form. For details on the use of the holomorphic null curve and the associated EnneperWeierstrass representation as well as historical background we refer the reader to standard works on minimal surfaces, such as [19,38,47,50,55,58].

For the purposes of this paper, it is however useful to point out two obvious ways to construct new minimal surfaces from a given minimal surface $f: M \rightarrow \mathbb{R}^{3}$ and its holomorphic null curve $\Phi$ : firstly, multiplying $\Phi$ by $e^{-i \theta}, \theta \in \mathbb{R}$, one obtains the associated family of minimal surfaces $f_{\cos \theta, \sin \theta}=\operatorname{Re}\left(e^{-i \theta} \Phi\right)$ as the real parts of the holomorphic null curves $e^{-i \theta} \Phi$. The associated family of minimal surfaces was introduced by Bonnet [14], in the study of surfaces parametrised by a curvilinear coordinate. An interesting feature of the associated family is that it is an isometric deformation of minimal surfaces which preserves the Gauss map. The converse was shown by Schwarz [66]: if two simply-connected minimal surfaces are isometric, then, by a suitable rigid motion, they belong to the same associated family.

The second transformation, the so-called Goursat transformation [30], is given by any orthogonal matrix $\mathcal{A} \in \mathrm{O}(3, \mathfrak{C})$ : since $\mathcal{A}$ preserves the standard symmetric bilinear form on $\mathfrak{C}^{3}$, the holomorphic map $\mathcal{A} \Phi$ is a null curve, and $\operatorname{Re}(\mathcal{A} \Phi)$ is a minimal surface in $\mathbb{R}^{3}$. As pointed out by Pérez and Ros [58], an interesting special case is known as the LópezRos deformation. To show that any complete, embedded genus zero minimal surface with finite total curvature is a catenoid or a plane, López and Ros [48] used a deformation of the Weierstrass data which preserves completeness and finite total curvature. This López-Ros deformation has been later used in various aspects of minimal surface theory, e.g., in the study of properness of complete embedded minimal surfaces [51], the discussion of symmetries of embedded genus $k$-helicoids [1], and in an approach to the Calabi-Yau problem [28].

On the other hand, by the Ruh-Vilms theorem the Gauss map of a minimal surface is a harmonic map $N: M \rightarrow S^{2}$ from a Riemann surface $M$ into the 2-sphere [63]. Harmonic maps from Riemann surfaces into compact Lie groups and symmetric spaces, or more generally, between Riemannian manifolds, have been extensively studied in the past. Harmonic maps are critical points of the energy functional and include a wide range of examples such as geodesics, minimal surfaces, Gauss maps of surfaces with constant mean curvature and classical solutions to non-linear sigma models in the physics of elementary particles. Surveys on the remarkable progress in this topic may be found in $[23,24,31,36,56]$.

One of the big breakthroughs in the theory of harmonic maps was the observation from theoretical physicists that a harmonic map equation is an integrable system $[53,60,67]$ : The harmonicity condition of a map from a Riemann surface into a suitable space can be expressed as a Maurer-Cartan equation. This equation allows to introduce the spectral parameter to obtain the associated family of connections. The condition for the map to be harmonic is then expressed by the condition that every connection in the family is a flat connection. This way, the harmonic map equation can be formulated as a Lax equation with parameter. Starting with the work of Uhlenbeck [71] integrable systems methods have been highly successful in the geometric study of harmonic maps from Riemann surfaces into suitable spaces, e.g., $[3,5,21,37,70,72]$. In particular, the theory can be used to describe the moduli spaces of surface classes which are given in terms of a harmonicity condition, such as constant mean curvature 
surfaces in $\mathbb{R}^{3}$ or $S^{3}$, e.g., [10,32,59], minimal surfaces in $S^{2} \times \mathbb{R}$, e.g., [33], Hamiltonian Stationary Lagrangians, e.g., [35,46], and Willmore surfaces, e.g., [4,11,34,64], and related surface classes such as isothermic surfaces, e.g., [2,6,18].

We recall the methods of integrable systems which are relevant for our paper: given a $\mathbb{C}_{*}$-family of flat connections $d_{\lambda}$ of the appropriate form, one can construct a harmonic map from it. In particular, the associated family $d_{\lambda}$ of flat connections of a harmonic map gives an element of the associated family of harmonic maps by, up to a gauge by a $d_{\mu}$-parallel endomorphism, using the family $d_{\mu \lambda}$ for some fixed $\mu \in \mathbb{C}_{*}$. The dressing operation was introduced by Uhlenbeck and Terng [70,71]: as pointed out to us by Burstall, in the case of a harmonic map $N: M \rightarrow S^{2}$ the dressing is given by a gauge $\hat{d}_{\lambda}=r_{\lambda} \cdot d_{\lambda}$ of $d_{\lambda}$ by a $\lambda$-dependent dressing matrix $r_{\lambda}$ [9]. The dressing of $N$ is then the harmonic map $\hat{N}$ that has $\hat{d}_{\lambda}$ as its associated family of flat connections. In general, it is hard to find explicit dressing matrices and compute the resulting harmonic map. However, if $r_{\lambda}$ has a simple pole $\mu \in \mathbb{C}_{*}$ and is given by a $d_{\mu}$-parallel bundle, then the so-called simple factor dressing can be computed explicitly, e.g., $[9,20,70]$.

Parallel bundles of the associated family of flat connections also play an important role in Hitchin's classification of harmonic tori in terms of spectral data [37], and in applications of his methods to constant mean curvature and Willmore tori, e.g., [59,64]. The holonomy representation of the family $d_{\lambda}$ with respect to a chosen base point on the torus is abelian and hence has simultaneous eigenlines. From the corresponding eigenvalues one can define the spectral curve $\Sigma$, a hyperelliptic curve over $\mathbb{C P}^{1}$ (which is independent of the chosen base point), together with a holomorphic line bundle over $\Sigma$, given by the eigenlines of the holonomy (these depend on the base point, and sweep out a subtorus of the Jacobian of the spectral curve). Conversely, the spectral data can be used to construct the harmonic tori in terms of theta-functions on the spectral curve $\Sigma$. This idea can be extended to a more general notion [68] of a spectral curve for conformal tori $f: T^{2} \rightarrow S^{4}$. Geometrically, this multiplier spectral curve arises as a desingularisation of the set of all Darboux transforms of $f$ where one uses a generalisation of the notion of Darboux transforms for isothermic surfaces to conformal surfaces [13].

As mentioned above, by the Ruh-Vilms theorem the Gauss map of a minimal immersion $f: M \rightarrow \mathbb{R}^{3}$ is harmonic, and thus, the various operations discussed above can be applied to its Gauss map. However, as opposed to the case of an immersion with constant non-vanishing mean curvature, the Gauss map does not uniquely determine the minimal surface. Thus, although the associated family and the dressing operation for the harmonic Gauss map of a minimal surface can be defined [22], the investigation of minimal surfaces with these dressed harmonic Gauss maps complicates. On the other hand, Meeks, Pérez and Ros [49,52], use algebro-geometric solutions to the $\mathrm{KdV}$ equation to show that the only properly embedded minimal planar domains with infinite topology are the Riemann minimal examples. The same Lamé potentials appear in the study of the spectral curve of an Euclidean minimal torus with two planar ends and translational periods [12]. This indicates that applying integrable system methods may lead to a further development of minimal surface theory. Conversely, getting a better understanding of the special case of minimal surfaces may also give insights into the more general methods from integrable systems.

The aim of our paper is to provide further evidence that concepts on minimal surfaces may in fact be special cases of the harmonic map theory: the López-Ros deformation is a special case of a simple factor dressing of a minimal surface.

To avoid the issue that a minimal surface is not uniquely determined by its Gauss map, we will work with the conformal Gauss map which determines a minimal surface in 3-space uniquely. Since minimal surfaces are Willmore the conformal Gauss map is harmonic, too. 
We will briefly recall the construction of the associated families $d_{\lambda}$ and $d_{\lambda}^{S}$ of flat connections for both the harmonic Gauss map $N$ and the conformal Gauss map $S$ of a minimal surface in our setup. Both are closely related: parallel sections of $d_{\lambda}^{S}$ can be expressed in terms of parallel sections of $d_{\lambda}$ and generalisations $f_{p, q}, p, q \in S^{3}$, of the associated family of minimal surfaces $f_{\cos \theta, \sin \theta}$. It turns out that this new family $f_{p, q}$, the right-associated family, is in fact a family of minimal surfaces in 4-space which contains the classical associated family. In view of this natural appearance of minimal surfaces in 4-space, we will develop our theory more generally for minimal surfaces in 4-space and restrict to the case of minimal surfaces in 3-space when appropriate. As in the case of a harmonic map $N: M \rightarrow S^{2}$ one can define the associated family of harmonic maps of the harmonic conformal Gauss map of a Willmore surface [8]. In the case of a minimal surface, we show that the harmonic maps in the associated family of the conformal Gauss map are indeed the conformal Gauss maps of the associated family of minimal surfaces.

Moreover, due to the harmonicity of the conformal Gauss map of a Willmore surface, a dressing operation on Willmore surfaces can be defined [4]. In particular, for the most simple dressing operation given by a dressing matrix with a simple pole, the so-called simple factor dressing, the new harmonic map can be computed explicitly and is the conformal Gauss map of a new Willmore surface in the 4-sphere [4,43].

In the case of a minimal surface $f: M \rightarrow \mathbb{R}^{4} \subset S^{4}$ we only consider simple factor dressings which preserve the Euclidean structure and show that in this case, the simple factor dressing of the conformal Gauss map of $f$ is indeed the conformal Gauss map of a minimal surface in 4-space. In fact, the simple factor dressing can be given explicitly in terms of the minimal surface $f$, its conjugate and the parameters $(\mu, m, n)$ where $\mu \in \mathbb{C} \backslash\{0\}$ is the pole of the simple factor dressing, and $m, n \in S^{3}$ determine the $d_{\mu}^{S}$-stable bundle which is needed in the definition of the dressing matrix. Even for surfaces in 3-space, the simple factor dressing will in general give surfaces in 4-space. However, for $n=m$, the simple factor dressing of a minimal surface $f: M \rightarrow \mathbb{R}^{3}$ will be in 3-space and the Gauss map of a simple factor dressing is the simple factor dressing of the Gauss map of $f$. In the simplest case when $n=m=1$ and $\mu \in \mathbb{R}$ the simple factor dressing is the minimal surface

$$
f^{\mu}=\left(\begin{array}{c}
f_{1} \\
f_{2} \cosh s-f_{3}^{*} \sinh s \\
f_{3} \cosh s+f_{2}^{*} \sinh s
\end{array}\right)
$$

where $s=-\ln |\mu|$ and $f_{l}, f_{l}^{*}$ are the coordinate functions of $f$ and a conjugate $f^{*}$ of $f$. In this case, we see immediately that $f^{\mu}$ is a Goursat transformation of $f$ with holomorphic null curve $\mathcal{L}^{\mu} \Phi$ where $\Phi=f+\mathbf{i} f^{*}$ is the holomorphic null curve of $f$ and

$$
\mathcal{L}^{\mu}=\left(\begin{array}{ccc}
1 & 0 & 0 \\
0 & \cosh s & \mathbf{i} \sinh s \\
0 & -\mathbf{i} \sinh s & \cosh s
\end{array}\right) \in \mathrm{O}(3, \mathfrak{C}) .
$$

Indeed, we prove more generally that every simple factor dressing of a minimal surface $f: M \rightarrow \mathbb{R}^{4}$ with parameters $(\mu, m, n)$ is a Goursat transformation. In particular, we show that this implies that the simple factor dressing preserves completeness. If the Goursat transform is single-valued on $M$ then finite total curvature is preserved, too.

In the case when $m=n \in S^{3}$, the orthogonal matrix of the Goursat transformation is given as $\mathcal{R}_{m, m} \mathcal{L}^{\mu} \mathcal{R}_{m, m}^{-1} \in \mathrm{O}(3, \mathfrak{C})$ where the rotation matrix $\mathcal{R}_{m, m}$ in 3-space is given by $m \in S^{3} \subset \mathbb{R}^{4}$ : decomposing $m=(\cos \theta, q \sin \theta)$ with $q \in \mathbb{R}^{3},\|q\|=1$, the matrix $\mathcal{R}_{m, m}$ is the rotation along the axis given by $q$ about the angle $2 \theta$. In other words, the simple factor 
dressing in $\mathbb{R}^{3}$ with parameters $(\mu, m, m)$ is obtained from the simple factor dressing (1) with parameter $\mu$ applied to the (inverse of the) rotation given by $m$.

The López-Ros deformation of a minimal surface $f: M \rightarrow \mathbb{R}^{3}$ is usually given in terms of the Weierstrass data. We recall an explicit form of the López-Ros deformation in terms of the minimal surface and its conjugate surface: the López-Ros deformation $f_{\sigma}$ of $f$ with parameter $\sigma \in \mathbb{R}, \sigma>0$, is indeed given by

$$
f_{\sigma}=\left(\begin{array}{c}
f_{1} \cosh s-f_{2}^{*} \sinh s \\
f_{2} \cosh s+f_{1}^{*} \sinh s \\
f_{3}
\end{array}\right)
$$

where $s=\ln \sigma$. In other words, since

$$
f_{\sigma}=\operatorname{Re}\left(\mathcal{R}_{m, m} \mathcal{L}^{\mu} \mathcal{R}_{m, m}^{-1} \Phi\right)
$$

with $\mu=-\frac{1}{\sigma}$ and $m=\frac{1}{2}(1,-1,-1,-1) \in S^{3}$, the Lopez-Ros deformation with parameter $\sigma$ is the simple factor dressing of $f$ with parameters $(\mu, m, m)$.

We investigate the periods of the simple factor dressings in terms of the periods of the holomorphic null curve, and give conditions on the parameters $(\mu, m, n)$ for a simple factor dressing to be single-valued. We discuss the end behaviour of the simple factor dressing on minimal surfaces in 3-space with finite total curvature ends: the simple factor dressing preserves planar ends for all parameters and, due to the special form of the Goursat transformation, catenoidal ends if the parameters of the simple factor dressings are chosen so that the simple factor dressing is single-valued.

We conclude the paper by demonstrating our results for various well-known minimal surfaces, including the catenoid, Richmond surfaces and Scherk's first surface. In particular, the simple factor dressings of the catenoid which are again periodic are reparametrisations of the catenoid if they are surfaces in 3-space. This immediately follows from our result that the simple factor dressing of a catenoidal end is catenoidal, provided the simple factor dressing is single-valued. Since planar ends are preserved for any parameters, all simple factor dressings of surfaces with one planar end have one planar end, too.

Using our closing conditions, we show that the López-Ros deformation of Scherk's first surface gives doubly-periodic minimal surfaces. Moreover, for any rational number $q>0$ we show that the simple factor dressing (1) with parameter $\mu=-\frac{1}{\sqrt{q}}$ is doubly-periodic, thus we obtain a family of new examples of doubly-periodic (non-embedded) minimal surfaces.

The authors would like to thank Wayne Rossman and Nick Schmitt for directing their attention towards the López-Ros deformation and the Goursat transformation. Parts of this research were conducted while the first author was visiting the Department of Mathematics at the University of Tsukuba and the OCAMI at Osaka University. The first author would like to thank the members of both institutions for their hospitality during her stay, and the University of Leicester for granting her study leave.

\section{Minimal surfaces}

We first recall some basic facts on minimal surfaces in Euclidean space which will be needed in the following whilst setting up our notation. Although we are mostly interested in minimal surfaces in $\mathbb{R}^{3}$, some of our transforms will be surfaces in $\mathbb{R}^{4}$. Therefore, we will study more generally minimal immersions in $\mathbb{R}^{4}$ and specialise to the case of minimal surfaces in 3-space when appropriate. 


\subsection{Minimal surfaces in $\mathbb{R}^{4}$}

Let $f: M \rightarrow \mathbb{R}^{4}$ be a conformal (branched) immersion from a Riemann surface $M$ into 4-space. If $f$ is minimal, then $f$ is harmonic, i.e.,

$$
d * d f=0
$$

where we put $* \omega(X)=\omega\left(J_{T M} X\right)$ for a 1-form $\omega \in \Omega^{1}(T M), X \in T M$. Here, $J_{T M}$ is the complex structure of the Riemann surface $M$, thus, $*$ is the negative Hodge star operator. In particular, $* d f$ is closed if $f$ is harmonic and there exists a conjugate surface $f^{*}$ on the universal cover $\tilde{M}$ of $M$, given up to translation by

$$
d f^{*}=-* d f .
$$

Note that $f^{*}$ is minimal, and so is the associated family (when lifting $f$ to the universal cover $\tilde{M})$, e.g. [25],

$$
f_{\cos \theta, \sin \theta}=f \cos \theta+f^{*} \sin \theta, \quad \theta \in \mathbb{R} .
$$

We say that a minimal surface $f: \tilde{M} \rightarrow \mathbb{R}^{4}$ is single-valued on $M$ if $f \circ \pi^{-1}: M \rightarrow \mathbb{R}^{4}$ is well-defined where $\pi: \tilde{M} \rightarrow M$ is the canonical projection of the universal cover $\tilde{M}$ to $M$. In this case, we will identify $f$ and $f \circ \pi^{-1}$ and write, in abuse of notation, from now on $f: M \rightarrow \mathbb{R}^{4}$.

We model Euclidean 4-space by the quaternions $\mathbb{R}^{4}=\mathbb{H}$, and the Euclidean 3-space in $\mathbb{R}^{4}$ by the imaginary quaternions $\mathbb{R}^{3}=\operatorname{Im} \mathbb{H}$. The conformality of an immersion $f: M \rightarrow \mathbb{R}^{4}$ gives [7, p. 10] the left and right normal $N, R: M \rightarrow S^{2}=\left\{n \in \operatorname{Im} \mathbb{H} \mid n^{2}=-1\right\}$ of $f$ by

$$
* d f=N d f=-d f R .
$$

Then the mean curvature vector $\mathcal{H}$ of $f: M \rightarrow \mathbb{R}^{4}$ satisfies [7, p. 39]

$$
\overline{\mathcal{H}} d f=\frac{1}{2}(* d R+R d R), \quad \text { or, equivalently, } \quad d f \overline{\mathcal{H}}=-\frac{1}{2}(* d N+N d N) .
$$

Since $\mathcal{H}$ is normal we have $N \mathcal{H}=\mathcal{H} R$. We put $H=-R \overline{\mathcal{H}}$ and denote by

$$
(d R)^{\prime}=\frac{1}{2}(d R-R * d R), \quad(d R)^{\prime \prime}=\frac{1}{2}(d R+R * d R)
$$

the $(1,0)$ and $(0,1)$-part of $d R$ with respect to the complex structure $R$. Then the equation of the mean curvature vector becomes

$$
H d f=(d R)^{\prime} .
$$

Similarly, there is also an equation for the mean curvature vector in terms of the left normal:

$$
d f H=\frac{1}{2}(d N-N * d N)=(d N)^{\prime} .
$$

Note that $f: M \rightarrow \mathbb{R}^{4}$ is minimal if and only if

$$
(d R)^{\prime}=0, \quad \text { or, equivalently, } \quad(d N)^{\prime}=0 .
$$

In other words, if $f$ is minimal then

$$
* d R=-R d R=d R R
$$


and $* d N=-N d N=d N N$ for the left and right normal of $f$. Thus, both $N$ and $R$ are quaternionic holomorphic sections [29] with respect to the induced quaternionic holomorphic structures on the trivial $\mathbb{H}$ bundle $\underline{\mathbb{H}}=M \times \mathbb{H}$. Note also that a map $R: M \rightarrow S^{2}$ is harmonic if and only if

$$
d(d R)^{\prime}=0 \quad \text { or, equivalently, } \quad d(d R)^{\prime \prime}=0 .
$$

In particular, both the left and right normal $N$ and $R$ of a minimal surface are conformal and harmonic.

Next, we observe that a conjugate surface $f^{*}$ of a minimal surface $f$ has the same left and right normal as $f$ since

$$
* d f^{*}=-*(* d f)=d f=* d f R=-d f^{*} R,
$$

and similarly $* d f^{*}=N d f^{*}$. Since $f$ is harmonic and $* d f=-d f^{*}$, the map

$$
\Phi=f+\mathbf{i} f^{*}: \tilde{M} \rightarrow \mathfrak{C}^{4}
$$

is a holomorphic curve in $\mathfrak{C}^{4}$, that is, $* d \Phi=\mathbf{i} d \Phi$. Here we use " $\mathbf{i}$ " to denote the complex structure of the complexification $\mathfrak{C}^{4}=\mathbb{R}^{4}+\mathbf{i} \mathbb{R}^{4}$ to avoid confusion with the imaginary quaternion $i$. If $f: M \rightarrow \mathbb{R}^{4}$ is a minimal conformal immersion, then the holomorphic curve

$$
\Phi=\left(\Phi_{0}, \Phi_{1}, \Phi_{2}, \Phi_{3}\right): \tilde{M} \rightarrow \mathfrak{C}^{4}
$$

is a null curve in $\mathfrak{C}^{4}$, i.e., $d \Phi_{0} \otimes d \Phi_{0}+d \Phi_{1} \otimes d \Phi_{1}+d \Phi_{2} \otimes d \Phi_{2}+d \Phi_{3} \otimes d \Phi_{3}=0$. In fact, every holomorphic null curve $\Phi: \tilde{M} \rightarrow \mathfrak{C}^{3}$ gives rise to a conformal minimal immersion by setting $f=\operatorname{Re}(\Phi): \tilde{M} \rightarrow \mathbb{R}^{3}$. Note that the holomorphic null curve of the associated family $f_{\cos \theta, \sin \theta}$ of a minimal surface $f$ is given by $\Phi_{\cos \theta, \sin \theta}=e^{-\mathbf{i} \theta} \Phi$ where $\Phi$ is the holomorphic null curve of $f$.

The Weierstrass data of $f$ is given by the meromorphic functions

$$
g_{1}=\frac{d \Phi_{3}}{d \Phi_{1}-\mathbf{i} d \Phi_{2}}, \quad g_{2}=\frac{d \Phi_{0}}{d \Phi_{1}-\mathbf{i} d \Phi_{2}}
$$

and the holomorphic 1-form

$$
\omega=d \Phi_{1}-\mathbf{i} d \Phi_{2}
$$

Conversely, let $g_{1}, g_{2}: M \rightarrow \mathfrak{C} \cup\{\infty\}$ be meromorphic functions and $\omega$ a holomorphic 1 -form. Assume that if $m$ is the maximum order of poles of $g_{1}, g_{2}$, and $g_{1}^{2}+g_{2}^{2}$ at $p \in M$ then $\omega$ has a zero at $p$ of order at least $m$. Then $\left(g_{1}, g_{2}, \omega\right)$ gives rise [39] to a holomorphic null curve $\Phi$, and thus a minimal surface $f=\operatorname{Re}(\Phi): \tilde{M} \rightarrow \mathbb{R}^{4}$, via

$$
\Phi=\int\left(g_{2} \omega, \frac{1}{2}\left(1-g_{1}^{2}-g_{2}^{2}\right) \omega, \frac{\mathbf{i}}{2}\left(1+g_{1}^{2}+g_{2}^{2}\right) \omega, g_{1} \omega\right) .
$$

Our choice of Enneper-Weierstrass representation is so that it specialises to the standard Enneper-Weierstrass representation in $\mathbb{R}^{3}$ whenever $f: \tilde{M} \rightarrow \mathbb{H}$ is a minimal surface with $\operatorname{Re}(f)=0$. We allow $f$ to be branched which happens when the order of $\omega$ at $p$ is bigger than the maximum order of poles of $g_{1}, g_{2}$, and $g_{1}^{2}+g_{2}^{2}$ at $p \in M$. Note that $f$ is in general only defined on the universal cover $\tilde{M}$ of $M$.

The Gauss map $G: M \rightarrow \mathrm{Gr}_{2}\left(\mathbb{R}^{4}\right)$ of $f$ is a map into the Grassmannian of oriented two planes in $\mathbb{R}^{4}$. In our case, it is locally given by $\varphi d z=d \Phi$ where the two-plane $G(p)$ in $\mathbb{R}^{4}$ is spanned by $\operatorname{Re} \varphi, \operatorname{Im} \varphi$ at $p$ since $d \Phi=d f+\mathbf{i} d f^{*}=d f-\mathbf{i} * d f$. But $\operatorname{Gr}_{2}\left(\mathbb{R}^{4}\right)=$ 
$\mathfrak{C} \mathbb{P}^{1} \times \mathfrak{C P}^{1}$ and the Gauss map $G$ can be identified [57] with the two meromorphic functions $G_{1}, G_{2}: M \rightarrow \mathfrak{C}:$

$$
G_{1}=\frac{\varphi_{3}-\mathbf{i} \varphi_{0}}{\varphi_{1}-\mathbf{i} \varphi_{2}}=g_{1}-\mathbf{i} g_{2}, \quad G_{2}=\frac{\varphi_{3}+\mathbf{i} \varphi_{0}}{\varphi_{1}-\mathbf{i} \varphi_{2}}=g_{1}+\mathbf{i} g_{2} .
$$

Indeed, stereographic projections of $G_{1}$ and $G_{2}$ give the left and right normals $N$ and $R$ by

$$
\begin{aligned}
& N=\frac{1}{1+\left|G_{1}\right|^{2}}\left(2 \operatorname{Re} G_{1}, 2 \operatorname{Im} G_{1},\left|G_{1}\right|^{2}-1\right), \\
& R=\frac{1}{1+\left|G_{2}\right|^{2}}\left(2 \operatorname{Re} G_{2}, 2 \operatorname{Im} G_{2},\left|G_{2}\right|^{2}-1\right) .
\end{aligned}
$$

To verify this, we note that $\Phi$ can be expressed in terms of $G_{1}, G_{2}, \omega$ as

$$
d \Phi=\varphi d z=\frac{\omega}{2}\left(\mathbf{i}\left(G_{1}-G_{2}\right), 1-G_{1} G_{2}, \mathbf{i}\left(1+G_{1} G_{2}\right), G_{1}+G_{2}\right) .
$$

Since $\varphi=f_{x}-\mathbf{i} f_{y}$ in the conformal coordinate $z=x+i y$, we obtain $f_{x}=$ $\frac{1}{2}(\varphi+\bar{\varphi}), f_{y}=\frac{\mathbf{i}}{2}(\varphi-\bar{\varphi})$, and it is a straight forward computation to verify that $N=\frac{1}{1+\left|G_{1}\right|^{2}}\left(2 \operatorname{Re} G_{1}, 2 \operatorname{Im} G_{1},\left|G_{1}\right|^{2}-1\right)$ and $R=\frac{1}{1+\left|G_{2}\right|^{2}}\left(2 \operatorname{Re} G_{2}, 2 \operatorname{Im} G_{2},\left|G_{2}\right|^{2}-1\right)$ satisfy $N f_{x}=-f_{x} R=f_{y}$ which are the defining equations (2) of the left and right normal of $f$.

Finally we recall the following result due to Chern and Osserman:

Theorem 2.1 ([17,54]) Let $f: M \rightarrow \mathbb{R}^{4}$ be a complete (branched) minimal immersion with holomorphic null curve $\Phi: M \rightarrow \mathfrak{C}^{4}$.

Then $f$ has finite total curvature if and only if $M$ is conformally equivalent to a compact Riemann surface $\bar{M}$ punctured at finitely many points $p_{1}, \ldots, p_{r}$ such that $d \Phi$ extends meromorphically into punctures $p_{i}$.

\subsection{Minimal surfaces in $\mathbb{R}^{3}$}

We identify the Euclidean 3-space with the imaginary quaternions $\mathbb{R}^{3}=\operatorname{Im} \mathbb{H}$. If $f: M \rightarrow$ $\mathbb{R}^{3}$ is conformal then the left and right normal coincide and are given by the Gauss map $N: M \rightarrow S^{2}$ of $f$. In this case, the function $H$ given in (4) is real-valued, and indeed, $H$ is the mean curvature function of $f$. For a minimal immersion in $\mathbb{R}^{3}$, the Gauss map is thus both harmonic and conformal, that is,

$$
* d N=-N d N, \quad \text { and } \quad d(d N)^{\prime}=0,
$$

where as before $(d N)^{\prime}=\frac{1}{2}(d N-N * d N)$ is the $(1,0)$-part of $d N$ with respect to the complex structure $N$.

The holomorphic null curve

$$
\Phi=f+\mathbf{i} f^{*}: \tilde{M} \rightarrow \mathfrak{C}^{3}
$$

gives the Weierstrass data $(g, \omega)$ of $f$ as

$$
\omega=d \Phi_{1}-\mathbf{i} d \Phi_{2} \quad \text { and } \quad g=\frac{d \Phi_{3}}{d \Phi_{1}-\mathbf{i} d \Phi_{2}},
$$

where $\Phi_{l}$ are the coordinates of $\Phi=\left(\Phi_{1}, \Phi_{2}, \Phi_{3}\right)$. 
Conversely, a meromorphic $g$ and a holomorphic 1-form $\omega$, such that if $g$ has a pole of order $m$ at $p$ then $\omega$ has a zero of order at least $2 m$, give a minimal surface $f: \tilde{M} \rightarrow \mathbb{R}^{3}$ as $f=\operatorname{Re}(\Phi)$ where $\Phi$ is given by the Enneper-Weierstrass representation $[27,73]$,

$$
\Phi=\int\left(\frac{1}{2}\left(1-g^{2}\right) \omega, \frac{\mathbf{i}}{2}\left(1+g^{2}\right) \omega, g \omega\right) .
$$

For convenience, we also will occasionally use in the case of a surface in $\mathbb{R}^{3}$ the Weierstrass data $(g, d h)$ given in terms of the height differential $d h=g \omega$. Written in terms of $(g, d h)$ the Enneper-Weierstrass representation becomes

$$
\Phi=\int\left(\frac{1}{2}\left(\frac{1}{g}-g\right), \frac{\mathbf{i}}{2}\left(\frac{1}{g}+g\right), 1\right) d h .
$$

The Gaussian curvature of $f$ is given in terms of the Weierstrass data [41] as

$$
K=-\left(\frac{2}{|g|+\frac{1}{|g|}}\right)^{4}\left|\frac{d \log g}{d h}\right|^{2} .
$$

and the Gauss map is given by $g$ via stereographic projection

$$
N=\frac{1}{|g|^{2}+1}\left(2 \operatorname{Re} g, 2 \operatorname{Im} g,|g|^{2}-1\right) .
$$

Note that if we consider a minimal surface $f: M \rightarrow \mathbb{R}^{3}$ as a surface in 4-space, that is $f: M \rightarrow \mathbb{H}$ with $\operatorname{Re}(f)=0$, then its Weierstrass data in $\mathbb{R}^{4}$ is under our choices given by $\left(g_{1}=g, g_{2}=0, \omega\right)$ and its holomorphic null curve $\int\left(0, \frac{1}{2}\left(1-g^{2}\right) \omega, \frac{\mathbf{i}}{2}\left(1+g^{2}\right) \omega, g \omega\right)$ in $\mathfrak{C}^{4}$ is given by the embedding of the curve $\Phi$ into $\mathfrak{C}^{4}$. Moreover, we see that in this case the Gauss map $G=\left(G_{1}, G_{2}\right): M \rightarrow S^{2} \times S^{2}$ takes values in the diagonal of $S^{2} \times S^{2}$ and is given by $G_{1}=G_{2}=g$ which is by $[39,69]$ the condition for the Enneper-Weierstrass representation to take values in 3-space.

As before, complete minimal immersions of finite total curvature can be characterised by the holomorphic null curve $\Phi$ :

Theorem 2.2 ([57]) Let $f: M \rightarrow \mathbb{R}^{3}$ be a complete (branched) minimal immersion with holomorphic null curve $\Phi: M \rightarrow \mathfrak{C}^{3}$.

Then $f$ has finite total curvature if and only if $M$ is conformally equivalent to a compact Riemann surface $\bar{M}$ punctured at finitely many points $p_{1}, \ldots, p_{r}$ such that $d \Phi$ extends meromorphically into punctures $p_{i}$.

We will now give a description of embedded finite total curvature ends in terms of the holomorphic null curve. Although the result seems to be known for vertical ends, we include the argument for completeness.

Theorem 2.3 Let $f: M \rightarrow \mathbb{R}^{3}$ be a minimal surface with complete end at $p$. Let $z$ be a conformal coordinate of $M$ at the end $p$ which is defined on a punctured disc $D_{*}=D \backslash\{0\}$ and is centered at $p$.

Then the following statements are equivalent:

(i) $f$ has an embedded finite total curvature end at $p$.

(ii) $d \Phi$ has order -2 at $z=0$ and $\operatorname{res}_{z=0} d \Phi$ is real.

If $\operatorname{res}_{z=0} d \Phi=0$ then the end is planar, otherwise, it is catenoidal. 
Here, $\Phi=\left(\Phi_{1}, \Phi_{2}, \Phi_{3}\right)$ is the holomorphic null curve of $f$ and $\operatorname{ord}_{z=0} d \Phi$ is the minimum of $\operatorname{ord}_{z=0} d \Phi_{i}$ for $i=1,2,3$.

Proof We first assume that the end is vertical. By [38] an embedded complete finite total curvature vertical end has logarithmic growth $\alpha \in \mathbb{R}$ satisfying $\operatorname{res}_{z=0} d \Phi=-(0,0,2 \pi \alpha)$. The end is planar if $\alpha=0$ and catenoidal otherwise.

Moreover, if $f$ has a catenoidal end then the Gauss map $g$ of $f$ has a simple pole or zero, and the height differential $d h$ has a simple pole. If $f$ has a planar end then $g$ has a pole or zero of order $m>1$ and $d h$ has a zero of order $m-2$. In both cases, (6) shows that $\operatorname{ord}_{z=0} d \Phi=-2$.

If the end is not vertical, we can apply a rotation $\mathcal{R} \in \mathrm{SO}(3, \mathbb{R})$ on $f$ to obtain a minimal surface $\tilde{f}=\mathcal{R} f$ with a vertical end. Then the holomorphic null curve of $\tilde{f}$ is $\tilde{\Phi}=\mathcal{R} \Phi$ and thus, $\operatorname{ord}_{z=0} d \Phi=\operatorname{ord}_{z=0} d \tilde{\Phi}=-2$. Moreover, the residues at $z=0$ vanish at a planar end, whereas $\operatorname{res}_{z=0} d \Phi=\mathcal{R}^{-1} \operatorname{res}_{z=0} d \tilde{\Phi}$ is real for a catenoidal end.

Conversely, we will show that if $\operatorname{ord}_{z=0} d \Phi=-2$ and $\operatorname{res}_{z=0} d \Phi$ is real, then we can assume $f$ has an embedded vertical end at $p$ and its Gauss map $g$ has a pole or zero of order $m \geq 1$ and $\operatorname{ord}_{z=0} d h=m-2$ holds for the height differential. From this we conclude that the end has finite total curvature.

If $\operatorname{res}_{z=0} d \Phi=(0,0,0)$ then we can rotate the end so that we have a vertical end without changing the order and the residues of $d \Phi$ at the end. In particular, we can assume that the Gauss map $g$ has a pole or zero at $p$ of order $m \geq 1$. Since the residue of $d \Phi$ at $z=0$ vanishes, we see that $d h$ cannot have a simple pole. But then ord ${ }_{z=0} d \Phi=-2$ implies that $d h$ is holomorphic with $\operatorname{ord}_{z=0} d h=m-2, m \geq 2$, so that with the Enneper-Weierstrass representation (6)

$$
d \Phi=\left(\begin{array}{c}
\frac{c_{-2}}{z^{2}}+b_{0}+\ldots \\
i \frac{c_{-2}}{z^{2}}+c_{0}+\ldots \\
a_{m-2} z^{m-2}+a_{m-1} z^{m-1}+\ldots
\end{array}\right) d z, \quad c_{-2}, a_{m-2} \neq 0 .
$$

After possible translation, we thus have

$$
\Phi(z)=\left(\begin{array}{c}
-\frac{c_{-2}}{z}+\ldots \\
-i \frac{c_{-2}}{z}+\ldots \\
\frac{a_{m-2}}{m-1} z^{m-1}+\ldots
\end{array}\right) .
$$

Consider the set $C_{r}=\left\{z \in D_{*} \mid\|f(z)\|=r\right\}$ where $f=\operatorname{Re}(\Phi)$. Since the end is at $z=0$, we have that $z \in C_{r}$ tends to 0 for $r \rightarrow \infty$. In particular, the asymptotic behaviour of $\psi_{r}=\frac{f}{r}: C_{r} \rightarrow S^{2}$ is governed by

$$
\psi_{r} \sim \frac{|z|}{\left|c_{-2}\right|}\left(\begin{array}{c}
\operatorname{Re}\left(-\frac{c_{-2}}{z}\right) \\
\operatorname{Im}\left(\frac{c_{-2}}{z}\right) \\
0
\end{array}\right) .
$$

From [40] we know that $\psi_{r}$ converges to a horizontal circle with multiplicity when $r \rightarrow \infty$ and that the end is embedded if the multiplicity is one. From the above asymptotic behaviour we see that the multiplicity of $\lim _{r \rightarrow \infty} \psi_{r}$ is indeed one. Hence, the end is embedded but then (8) shows that $p$ is a planar end.

If the order of $d \Phi=-2$ and $\operatorname{res}_{z=0} d \Phi \neq 0$ is real then we can rotate the end so that $\operatorname{res}_{z=0} d \Phi=-(0,0,2 \pi \alpha)$ for some $\alpha \in \mathbb{R}_{*}=\mathbb{R} \backslash\{0\}$. Then the order of $d h$ is either -1 or -2 . 
We first show that the end is vertical by contradiction: if $\mathrm{g}$ has neither a zero nor a pole at the end, then $\operatorname{ord}_{z=0} g d h=\operatorname{ord}_{z=0} \frac{d h}{g}=\operatorname{ord}_{z=0} d h$. Using $\operatorname{ord}_{z=0} d \Phi=-2$ and the Enneper-Weierstrass representation (6), the order of $d h$ at the end is -2 . We write

$$
d h=\left(\frac{a_{-2}}{z^{2}}-\frac{\alpha}{z}+a_{0}+a_{1} z+\ldots\right) d z, \quad g=b_{0}+b_{1} z+\ldots
$$

where $a_{-2}, b_{0} \neq 0$. Since

$$
g d h=\left(\frac{a_{-2} b_{0}}{z^{2}}+\frac{-\alpha b_{0}+a_{-2} b_{1}}{z}+\ldots\right) d z, \quad \frac{d h}{g}=\left(\frac{a_{-2}}{b_{0} z^{2}}-\frac{\alpha b_{0}+a_{-2} b_{1}}{b_{0}^{2} z}+\ldots\right) d z
$$

and $\operatorname{res}_{z=0} d \Phi_{1}=\operatorname{res}_{z=0} d \Phi_{2}=0$, we have

$$
\left(-\alpha b_{0}+a_{-2} b_{1}\right)+\frac{\alpha b_{0}+a_{-2} b_{1}}{b_{0}^{2}}=0, \quad\left(-\alpha b_{0}+a_{-2} b_{1}\right)-\frac{\alpha b_{0}+a_{-2} b_{1}}{b_{0}^{2}}=0 .
$$

Hence $\alpha b_{0}=0$ which contradicts $\alpha \neq 0$ and $b_{0} \neq 0$. Therefore, $g$ has a zero or a pole at the end, but then the end has vertical normal, the zero or pole of $g$ is simple and $d h$ has a simple pole at $z=0$, that is, the holomorphic null curve $\Phi$ can be written as

$$
d \Phi=\left(\begin{array}{c}
\frac{c_{-2}}{z^{2}}+b_{0}+\ldots \\
i \frac{c_{-2}}{z^{2}}+c_{0}+\ldots \\
\frac{a_{-1}}{z}+a_{0}+\ldots
\end{array}\right) d z, \quad c_{-2}, a_{-1} \neq 0 .
$$

Since $\operatorname{res}_{z=0} d \Phi=(0,0,-2 \pi \alpha)$ we have $a_{-1}=-2 \pi \alpha \in \mathbb{R}$ and, after possibly translation,

$$
f=\operatorname{Re} \Phi=\left(\begin{array}{c}
-\operatorname{Re}\left(\frac{c_{-2}}{z}+\ldots\right) \\
\operatorname{Im}\left(\frac{c_{-2}}{z}+\ldots\right) \\
\left.a_{-1} \log |z|+\operatorname{Re}\left(a_{0} z\right)+\ldots\right)
\end{array}\right) .
$$

Let as before $C_{r}=\left\{z \in D_{*} \mid\|f(z)\|=r\right\}$ then the asymptotic behaviour of $\psi_{r}=\frac{f}{r}$ : $C_{r} \rightarrow S^{2}$ is determined by

$$
\psi_{r} \sim \frac{1}{\sqrt{\left(\frac{\left|c_{-2}\right|}{|z|}\right)^{2}+\left(a_{-1} \log |z|\right)^{2}}}\left(\begin{array}{c}
-\operatorname{Re}\left(\frac{c_{-2}}{z}\right) \\
\operatorname{Im}\left(\frac{c_{-2}}{z}\right) \\
a_{-1} \log |z|
\end{array}\right) .
$$

Since the multiplicity does not depend on $|z|$ we see that in the limit $r \rightarrow \infty$ the multiplicity is again 1, and the end is embedded by [40]. Following the arguments in the proof of [38, Prop. 2.1], $f$ is a graph over (the exterior of a bounded domain of) the $\left(f_{1}, f_{2}\right)$ plane with asymptotic behaviour

$$
f_{3}\left(f_{1}, f_{2}\right)=\alpha \log R+\beta+R^{-2}\left(\gamma_{1} f_{1}+\gamma_{2} f_{2}\right)+O\left(R^{-2}\right)
$$

for $R=\sqrt{f_{1}^{2}+f_{2}^{2}}$, and the end has finite total curvature [65] .

The López-Ros deformation of a minimal surface $f: M \rightarrow \mathbb{R}^{3}$ with Weierstrass data $(g, \omega)$ is [48] the minimal surface $f_{r}: \tilde{M} \rightarrow \mathbb{R}^{3}$ given by the new Weierstrass data $\left(r g, \frac{\omega}{r}\right)$ with $r \in \mathbb{R}_{*}$. Obviously this can be extended to a deformation $f_{\sigma}$ with complex parameter $\sigma \in \mathfrak{C}_{*}=\mathfrak{C} \backslash\{0\}$ by using the Weierstrass data $\left(\sigma g, \frac{\omega}{\sigma}\right)$. 
Since any matrix in $\mathrm{O}(3, \mathfrak{C})=\left\{\mathcal{A} \in \mathrm{GL}(3, \mathfrak{C}) \mid \mathcal{A}^{\mathrm{t}}=\mathcal{A}^{-1}\right\}$ preserves the standard symmetric bilinear form on $\mathfrak{C}^{3}$, we obtain new minimal surfaces via the Goursat transformation [30]: if $f: M \rightarrow \mathbb{R}^{3}$ is minimal with holomorphic null curve $\Phi=f+\mathbf{i} f^{*}$ then $\mathcal{A} \Phi$ is again a holomorphic null curve and $\operatorname{Re}(\mathcal{A} \Phi)$ is a minimal surface in $\mathbb{R}^{3}$.

As pointed out by Pérez and Ros [58], the López-Ros deformation is a special case of the Goursat transformation:

Theorem 2.4 The López-Ros deformation $f_{\sigma}$ with complex parameter $\sigma=e^{s+\mathbf{i} t} \in \mathfrak{C}_{*}$ is given by

$$
f_{\sigma}=\left(\begin{array}{c}
\cos t\left(f_{1} \cosh s-f_{2}^{*} \sinh s\right)-\sin t\left(f_{2} \cosh s+f_{1}^{*} \sinh s\right) \\
\sin t\left(f_{1} \cosh s-f_{2}^{*} \sinh s\right)+\cos t\left(f_{2} \cosh s+f_{1}^{*} \sinh s\right) \\
f_{3}
\end{array}\right) .
$$

Proof Let $\Phi=f+\mathbf{i} f^{*}$ be the holomorphic null curve of $f$. Putting

$$
\tilde{f}=\left(\begin{array}{ccc}
\cos t & -\sin t & 0 \\
\sin t & \cos t & 0 \\
0 & 0 & 1
\end{array}\right)\left(\begin{array}{c}
f_{1} \cosh s-f_{2}^{*} \sinh s \\
f_{2} \cosh s+f_{1}^{*} \sinh s \\
f_{3}
\end{array}\right),
$$

a straightforward computation shows that $\tilde{f}=\operatorname{Re}\left(\mathcal{L}_{\sigma} \Phi\right)$ where the holomorphic map $\mathcal{L}_{\sigma} \Phi$,

$$
\mathcal{L}_{\sigma}=\left(\begin{array}{ccc}
\frac{1}{2}\left(\sigma+\frac{1}{\sigma}\right) & \frac{i}{2}\left(\sigma-\frac{1}{\sigma}\right) & 0 \\
-\frac{i}{2}\left(\sigma-\frac{1}{\sigma}\right) & \frac{1}{2}\left(\sigma+\frac{1}{\sigma}\right) & 0 \\
0 & 0 & 1
\end{array}\right) \in \mathrm{O}(3, \mathfrak{C})
$$

is a null curve in $\mathfrak{C}^{3}$. Thus, $\tilde{f}$ is a Goursat transformation of $f$. Indeed, if $(g, \omega)$ denotes the Weierstrass data of $f$ then the Weierstrass data of $\tilde{f}$ computes to

$$
\tilde{\omega}=d \tilde{\Phi}_{1}-\mathbf{i} d \tilde{\Phi}_{2}=\frac{\omega}{\sigma}
$$

and

$$
\tilde{g}=\frac{d \tilde{\Phi}_{3}}{d \tilde{\Phi}_{1}-\mathbf{i} d \tilde{\Phi}_{2}}=\sigma g .
$$

This shows that $\tilde{f}=f_{\sigma}$ is the López-Ros deformation of $f$ with parameter $\sigma \in \mathfrak{C}_{*}$.

\subsection{Willmore surfaces}

Using the one-point compactification of $\mathbb{R}^{4}$ we consider a conformal immersion $f: M \rightarrow \mathbb{R}^{4}$ as a conformal immersion into the 4 -sphere. We identify the 4 -sphere $S^{4}=\mathbb{H P}^{1}$ with the quaternionic projective line where the oriented Möbius transformations are given by $\mathrm{GL}(2, \mathbb{H})$. In particular, a map $f: M \rightarrow \mathbb{H} \mathbb{P}^{1}$ can be identified with a line subbundle $L \subset \underline{\mathbb{H}}^{2}=M \times \mathbb{H}^{2}$ of the trivial $\mathbb{H}^{2}$ bundle over $M$ whose fibers at $p \in M$ are given by

$$
L_{p}=f(p) .
$$

For an immersion $f: M \rightarrow \mathbb{R}^{4}$ the line bundle $L$ is given by

$$
L=\psi \mathbb{H}, \quad \text { where } \quad \psi=\left(\begin{array}{l}
f \\
1
\end{array}\right)
$$


when choosing the point at infinity as $\infty=\left(\begin{array}{l}1 \\ 0\end{array}\right) \mathbb{H} \in \mathbb{H} \mathbb{P}^{1}$. Oriented Möbius transformations on $\mathbb{R}^{4}=\mathbb{H}$ are given by

$$
v \mapsto(a v+b)(c v+d)^{-1} \text { with }\left(\begin{array}{ll}
a & b \\
c & d
\end{array}\right) \in \operatorname{GL}(2, \mathbb{H}) .
$$

In particular, the group of oriented Möbius transformations acts on the line bundle $L$ given by an immersion $f: M \rightarrow \mathbb{R}^{4}$ via $L \mapsto B L, B \in \mathrm{GL}(2, \mathbb{H})$. Every pair of unit quaternions $m, n \in S^{3}$ gives an element $\mathcal{R}_{m, n} \in \mathrm{SO}(4, \mathbb{R})$ by

$$
v \in \mathbb{H} \mapsto \mathcal{R}_{m, n} v=m v n^{-1} \in \mathbb{H},
$$

and conversely, every element of the special orthogonal group arises this way [16]. The corresponding action on the line bundle $L$ of an immersion $f: M \rightarrow \mathbb{R}^{4}$ is given by

$$
L \mapsto\left(\begin{array}{cc}
m & 0 \\
0 & n
\end{array}\right) L, \quad m, n \in S^{3} .
$$

Definition 2.5 ([7, p. 27]). The conformal Gauss map of a conformal immersion $f: M \rightarrow S^{4}$ is the unique complex structure $S$ on $\underline{\mathbb{H}}^{2}$ such that $S$ and $d S$ stabilise the line bundle $L$ of $f$ and its Hopf field $A$ is a 1 -form with values in $L$.

Here, the Hopf field $A$ of $S$ is the 1-form given by

$$
A=\frac{1}{4}(* d S+S d S)=\frac{1}{2}(* d S)^{\prime}
$$

where $(d S)^{\prime}=\frac{1}{2}(d S-S * d S)$ is the $(1,0)$-part of the derivative of $S$ with respect to the complex structure $S$.

In affine coordinates, the conformal Gauss map of a conformal immersion $f: M \rightarrow \mathbb{R}^{4}$ is given by the complex structure, see [7, p. 42],

$$
S=G\left(\begin{array}{cc}
N & 0 \\
-H & -R
\end{array}\right) G^{-1}, \quad G=\left(\begin{array}{cc}
1 & f \\
0 & 1
\end{array}\right)
$$

on the trivial bundle $\underline{\mathbb{H}}^{2}$ where $N, R$ are the left and right normal of $f$ and $H=-R \overline{\mathcal{H}}$ with mean curvature vector $\mathcal{H}$. Thus, we see that $S \psi=-\psi R$ and

$$
(d S) \psi=\psi(-d R+H d f) .
$$

Therefore, $S$ and $d S$ indeed stabilise the line bundle $L=\psi \mathbb{H}$ of $f$. In affine coordinates, the Hopf field computes [7, Prop 12, p. 42] to

$$
2 * A=G\left(\begin{array}{cc}
0 & 0 \\
\omega & \tau
\end{array}\right) G^{-1}
$$

with $2 \omega=d H+H * d f H+R * d H-H * d N$ satisfying

$$
* \omega=-\omega N+(d R)^{\prime \prime} H
$$

and

$$
\tau=(d R)^{\prime \prime}=\frac{1}{2}(d R+R * d R) .
$$

Thus, $A$ is indeed a 1 -form with values in $L$. Note that the Hopf field $A$ is holomorphic [7, p. 68] with im $A \subset L$. In particular, if $A \not \equiv 0$, the Hopf field $A$ gives the line bundle $L$ 
by holomorphically extending im $A$ into the isolated zeros of $A$. Therefore, when fixing the point at $\infty$, the immersion $f: M \rightarrow \mathbb{R}^{4}$ is uniquely determined by $A$.

Corollary 2.6 A conformal immersion $f: M \rightarrow \mathbb{R}^{4}$ is minimal if and only if the Hopf field A of the conformal Gauss map $S$ of $f$ satisfies

$$
2 * A=G\left(\begin{array}{cc}
0 & 0 \\
0 & d R
\end{array}\right) G^{-1}
$$

with $G=\left(\begin{array}{ll}1 & f \\ 0 & 1\end{array}\right)$

In particular, if $f: M \rightarrow \mathbb{R}^{4}$ is minimal then $* d R=-R d R$ and thus $d f \wedge d R=0$ by type arguments. Therefore, the Hopf field is harmonic, that is, $d * A=0$.

Theorem 2.7 ([26,62]) Let $f: M \rightarrow S^{4}$ be a conformal immersion with conformal Gauss map $S$ and Hopf field A. Then $f$ is Willmore if and only if $S$ is harmonic, that is, if and only if

$$
d * A=0 \text {. }
$$

From this characterisation of Willmore surfaces and the fact that the conformal Gauss map of a minimal surface is harmonic we see:

Corollary 2.8 Every minimal immersion in $\mathbb{R}^{4}$ is a Willmore surface in $\mathbb{R}^{4}$.

\section{Harmonic maps and their associated families of flat connections}

It is well-known [71] that a harmonic map gives rise to a family of flat connections. There are various transformations on harmonic maps whose new harmonic maps are build from parallel sections of the associated family of flat connections: e.g., the associated family, the simple factor dressing [70] and Darboux transforms [9,15]. In this paper, we investigate the links between the first two transformations, when applied to the left and right normals and to the conformal Gauss map of a minimal surface $f: M \rightarrow \mathbb{R}^{4}$. For the resulting Darboux transforms and their relation to the dressing we refer to [44].

\subsection{The harmonic right normal and its associated family}

We equip $\mathbb{H}$ with the complex structure $I$ which is given by the right multiplication by the unit quaternion $i$. This way, we identify $\mathbb{C}^{2}=(\mathbb{H}, I)$. It is worthwhile to point out that this complex structure $I$ differs from the complex structure $\mathbf{i}$ we used before. We will use the symbol $\mathbb{C}=\operatorname{span}_{\mathbb{R}}\{1, I\}$ to indicate that we use the complex structure $I$ whereas $\mathfrak{C}=\operatorname{span}_{\mathbb{R}}\{1, \mathbf{i}\}$.

With this at hand, the $\mathbb{C}_{*}$-family of flat connections of a harmonic map $R: M \rightarrow S^{2}$ on the trivial $\mathbb{C}^{2}$ bundle $\underline{\mathbb{C}}^{2}=\underline{\mathbb{H}}$ over $M$ can be written as, see for example $[9,15]$,

$$
d_{\lambda}=d+(\lambda-1) Q^{(1,0)}+\left(\lambda^{-1}-1\right) Q^{(0,1)},
$$

where $d$ is the trivial connection on $\underline{\mathbb{H}}, \lambda \in \mathbb{C}_{*}=\mathbb{C} \backslash\{0\}$, and $Q=-\frac{1}{2}(* d R)^{\prime \prime}=\frac{1}{4}(R d R-$ $* d R)$ is the Hopf field of $R$. Moreover, 


$$
Q^{(1,0)}=\frac{1}{2}(Q-I * Q), \quad Q^{(0,1)}=\frac{1}{2}(Q+I * Q)
$$

are the $(1,0)$ and $(0,1)$-parts of $Q$ with respect to the complex structure $I$.

In the situation when $R$ is the right normal of a minimal surface $f: M \rightarrow \mathbb{R}^{4}$ the map $R$ is conformal with $* d R=-R d R$, so that $2 * Q=d R$ and, using $R d R=-d R R$,

$$
Q^{(1,0)}=-\frac{1}{4} d R(I+R), \quad Q^{(0,1)}=\frac{1}{4} d R(I-R) .
$$

Recalling that $I$ operates from the right we obtain $d_{\lambda}=d+\alpha_{\lambda}$ with

$$
\alpha_{\lambda}=\frac{1}{2} d R(-R(a-1)+b),
$$

where $a=\frac{\lambda+\lambda^{-1}}{2}, b=I \frac{\lambda^{-1}-\lambda}{2}$. Since $-R d R=d R R$ and $a^{2}+b^{2}=1$, we see

$$
\alpha_{\lambda} \wedge \alpha_{\lambda}=\frac{1}{4} d R \wedge d R\left((a-1)^{2}+b^{2}\right)=\frac{1}{2} d R \wedge d R(1-a) .
$$

On the other hand,

$$
d \alpha_{\lambda}=\frac{1}{2} d R \wedge d R(a-1)
$$

and we showed that $d_{\lambda}=d+\alpha_{\lambda}$ is flat for all $\lambda \in \mathbb{C}_{*}$ in our special case when $* d R=-R d R$. The flatness of $d_{\lambda}$ for a general harmonic map $R$ is obtained from (5), that is, from $d * Q=0$.

We first note that for $\mu=1$ the connection $d_{\mu}=d$ is the trivial connection on $\underline{\mathbb{H}}$, and all parallel sections are constants. Thus, we will from now on assume that $\mu \neq 1$. Fix $\mu \in \mathbb{C} \backslash\{0,1\}$ and consider a $d_{\mu}$-parallel section $\beta \in \Gamma(\underline{\mathbb{H}})$, that is,

$$
0=d_{\mu} \beta=d \beta+\frac{1}{2} d R(-R \beta(a-1)+\beta b),
$$

where $a=\frac{\mu+\mu^{-1}}{2}, b=i \frac{\mu^{-1}-\mu}{2}$. Denoting by

$$
m=\frac{1}{2}(R \beta(a-1)-\beta b),
$$

we obtain with $R^{2}=-1$ and $a^{2}+b^{2}=1$ that

$$
R m+m \frac{b}{a-1}=\frac{1}{2} R(R \beta(a-1)-\beta b)+\frac{1}{2}(R \beta(a-1)-\beta b) \frac{b}{a-1}=\beta .
$$

We show that $m$ is constant: since $a^{2}+b^{2}=1, R d R=-d R R$ and $d \beta=d R m$ by (13) we have

$$
\begin{aligned}
2 d m & =d R \beta(a-1)+R d \beta(a-1)-d \beta b \\
& =d R \beta(a-1)-d R\left(R m+m \frac{b}{a-1}\right)(a-1)=0 .
\end{aligned}
$$

In particular, we have seen that every $d_{\mu}$-parallel section is given by

$$
\beta=R m+m \frac{b}{a-1}
$$

with $m \in \mathbb{H}$. Note that $\beta$ is a global parallel section of the trivial connection $d_{\mu}$ which is nowhere vanishing if $m \neq 0$ : If $R(p)=m \frac{b}{1-a} m^{-1}$ then 


$$
-1=R^{2}(p)=m \frac{b^{2}}{(1-a)^{2}} m^{-1}=m \frac{1+a}{1-a} m^{-1},
$$

where we used $a^{2}+b^{2}=1$. Therefore $-1=\frac{1+a}{1-a}$ which gives a contradiction.

Conversely, every $\beta$ given by the equation (15) is $d_{\mu}$-parallel. Since

$$
\frac{b}{a-1}=\frac{i(1+\mu)}{1-\mu}
$$

we can summarise:

Lemma 3.1 Let $f: M \rightarrow \mathbb{R}^{4}$ be minimal and $d_{\lambda}$ the associated family of the right normal $R$ of $f$. For $\mu \in \mathbb{C} \backslash\{0,1\}$ every (non-trivial) $d_{\mu}$-parallel section $\beta \in \Gamma(\underline{\mathbb{H}})$ is given by

$$
\beta=R m+m \frac{i(1+\mu)}{1-\mu}, \quad m \in \mathbb{H}_{*}=\mathbb{H} \backslash\{0\} .
$$

In a similar way, the associated family of the left normal of a minimal surface can be discussed.

Remark 3.2 Note that our choice of associated family differs from the associated family

$$
\hat{d}_{\lambda}=d+(\lambda-1) A_{R}^{(1,0)}+\left(\lambda^{-1}-1\right) A_{R}^{(0,1)}
$$

used in $[9,15]$ where $A_{R}=\frac{1}{2}(* d R)^{\prime}=\frac{1}{4}(* d R+R d R)$. However, since

$$
Q=\frac{1}{4}(R d R-* d R)=\frac{1}{4}(* d(-R)+(-R) d(-R))
$$

we see that our family of flat connections $d_{\lambda}$ is the associated family in $[9,15]$ of the harmonic map $-R$. Indeed, both families are gauge equivalent [15]. We choose the $d_{\lambda}$ family since it is closely related to the associated family of flat connections of the conformal Gauss map.

\subsection{The conformal Gauss map and its associated family}

Again, we identify $\mathbb{C}^{4}=\left(\mathbb{H}^{2}, I\right)$ where $I$ is given by right multiplication by the unit quaternion $i$. If the conformal Gauss map of a conformal immersion $f: M \rightarrow S^{4}$ is harmonic, that is $d * A=0$, by the same arguments as in the case of harmonic maps into the 2 -sphere, the $\mathbb{C}_{*}$-family of connections

$$
d_{\lambda}^{S}=d+(\lambda-1) A^{(1,0)}+\left(\lambda^{-1}-1\right) A^{(0,1)}, \quad \lambda \in \mathbb{C}_{*},
$$

is flat [43] on the trivial $\mathbb{C}^{4}$ bundle over $M$ where as before

$$
A^{(1,0)}=\frac{1}{2}(A-I * A) \quad \text { and } \quad A^{(0,1)}=\frac{1}{2}(A+I * A)
$$

denote the $(1,0)$ and $(0,1)$ parts of $A$ with respect to $I$.

We consider the case when $S$ is the conformal Gauss map of a minimal immersion in $\mathbb{R}^{4}$. We fix $\mu \in \mathbb{C}_{*}$ and compute all parallel sections of $d_{\mu}^{S}$. If $\mu=1$ then $d_{\mu}^{S}=d$ is trivial, and every constant section is parallel. Assume from now on that $\mu \neq 1$, and let

$$
e=\left(\begin{array}{l}
1 \\
0
\end{array}\right), \quad \psi=\left(\begin{array}{l}
f \\
1
\end{array}\right)
$$


and $L=\psi \mathbb{H}$ the line bundle of $f$. We denote by $\widetilde{\mathbb{H}}^{2}$ the pull back of the trivial $\mathbb{H}^{2}$ bundle under the canonical projection $\pi: \tilde{M} \rightarrow M$ of the universal cover $\tilde{M}$ to $M$. Since $e \mathbb{H} \oplus L=\underline{\mathbb{H}}^{2}$ every $d_{\mu}^{S}$-parallel section $\varphi \in \Gamma\left(\underline{\widetilde{\mathbb{H}}}^{2}\right)$ can be written as

$$
\varphi=e \alpha+\psi \beta
$$

where

$$
d \alpha=-d f \beta
$$

and

$$
d \beta=\frac{1}{2} d R(R \beta(a-1)-\beta b)
$$

with $a=\frac{\mu+\mu^{-1}}{2}, b=i \frac{\mu^{-1}-\mu}{2}$. Here we used that $* d R=-R d R=d R R$ by the minimality of $f$ and thus from (11) we see

$$
A^{(1,0)} \psi=-\frac{1}{4} \psi d R(R+i), \quad A^{(0,1)} \psi=-\frac{1}{4} \psi d R(R-i) .
$$

In particular, $\beta$ is a $d_{\mu}$-parallel section by (13), and by Lemma 3.1 it is given by

$$
\beta=R m+m \frac{i(1+\mu)}{1-\mu}
$$

with $m \in \mathbb{H}$. If $\beta=0$ is trivial, that is, $m=0$, then (20) shows that $\alpha$ is constant. For $m \neq 0$, we see that

$$
\alpha=-f^{*} m-f m \frac{i(1+\mu)}{1-\mu},
$$

is the general solution of (20) where $f^{*}$ is a conjugate minimal surface of $f$, that is,

$$
d f^{*}=-* d f=d f R .
$$

We summarise:

Proposition 3.3 Let $f: M \rightarrow \mathbb{R}^{4}$ be a minimal surface with conjugate surface $f^{*}$ and $d_{\lambda}^{S}$ the associated family of flat connections of the conformal Gauss map $S$ of $f$. For $\mu \in$ $\mathbb{C} \backslash\{0,1\}$ every $d_{\mu}^{S}$-parallel section is either a constant section $\varphi=e n, n \in \mathbb{H}$, or is given by $\varphi=e \alpha+\psi \beta \in \Gamma\left(\underline{\widetilde{\mathbb{H}}}^{2}\right)$ with

$$
\alpha=-f^{*} m-f m \frac{i(1+\mu)}{1-\mu}, \quad \beta=R m+m \frac{i(1+\mu)}{1-\mu}, \quad m \in \mathbb{H}_{*} .
$$

\section{The associated family of a minimal surface}

Motivated by our observation (22) that parallel sections of the associated family of flat connections of a minimal surface $f: M \rightarrow \mathbb{R}^{4}$ are given by a quaternionic linear combination of $f$ and its conjugate surface $f^{*}$, we now discuss a generalisation of the associated family of a minimal surface. This associated family is indeed given by the associated family of the harmonic conformal Gauss map. 


\subsection{The generalised associated family}

Given a minimal surface $f: M \rightarrow \mathbb{R}^{4}$ with left and right normal $N$ and $R$ respectively, we have seen that its conjugate surface $f^{*}: \tilde{M} \rightarrow \mathbb{R}^{4}$ has the same left and right normals $N$ and $R$ respectively since $d f^{*}=-* d f$.

In particular, the associated family $f_{\cos \theta, \sin \theta}=f \cos \theta+f^{*} \sin \theta$ can easily be extended to a family

$$
f_{p, q}=f p+f^{*} q: \tilde{M} \rightarrow \mathbb{R}^{4}
$$

with $p, q \in \mathbb{H},(p, q) \neq(0,0)$. Since $p+R q$ is a quaternionic holomorphic section it has only isolated zeros, and

$$
d f_{p, q}=d f(p+R q)
$$

shows that $f_{p, q}$ is a branched conformal immersion. The right normal $R$ of $f$ gives, away from the isolated zeros of $p+R q$, via

$$
R_{p, q}=(p+R q)^{-1} R(p+R q)
$$

the right normal of $f_{p, q}$ whereas the left normal $N$ of $f$ is the left normal

$$
N_{p, q}=N
$$

of $f_{p, q}$. Thus, by (4)

$$
d f_{p, q} H_{p, q}=\left(d N_{p, q}\right)^{\prime}=d N^{\prime}=0
$$

and $f_{p, q}$ is a (branched) minimal immersion. Similarly, we have a family of (branched) minimal immersions $f^{p, q}=p f+q f^{*},(p, q) \neq(0,0)$, with right normal $R^{p, q}=R$ and left normal $N^{p, q}=(p-q N) N(p-q N)^{-1}$.

Definition 4.1 Let $f: M \rightarrow \mathbb{R}^{4}$ be a minimal surface. The family of (branched) minimal immersions

$$
f_{p, q}=f p+f^{*} q: \tilde{M} \rightarrow \mathbb{R}^{4}, \quad p, q \in \mathbb{H},(p, q) \neq(0,0),
$$

where $f^{*}: \tilde{M} \rightarrow \mathbb{R}^{4}$ is a conjugate surface of $f$, is called the right associated family of $f$. The family of (branched) minimal immersion

$$
f^{p, q}=p f+q f^{*}: \tilde{M} \rightarrow \mathbb{R}^{4}, \quad p, q \in \mathbb{H}, \quad(p, q) \neq(0,0),
$$

is called the left associated family of $f$.

Note that for $p, q \in \mathbb{R},(p, q) \neq(0,0)$, we obtain the usual associated family of a minimal surface up to scaling. Moreover, $f_{p n, q n}=f_{p, q} n$ is given by a scaling of $f_{p, q}$ and an isometry on $\mathbb{R}^{4}$ for $n \in \mathbb{H}_{*}$.

Theorem 4.2 The right (left) associated family is a $S^{3}$-family of minimal surfaces in $\mathbb{R}^{4}$ which contains the classical associated family $f_{\cos \theta} \theta \sin \theta, \theta \in \mathbb{R}$, of minimal surfaces.

The right (left) associated family preserves the conformal class, and a surface $f_{p, q}$ (or $\left.f^{p, q}\right)$ is isometric to $f$ if and only if it is an element of the classical associated family, up to an isometry of $\mathbb{R}^{4}$. 
Proof It only remains to show that $f_{p, q}$ is isometric to $f$ if and only if $f_{p, q}=f_{n \cos \theta, n \sin \theta}$ for some $\theta \in \mathbb{R}$ and $n \in S^{3}$. Assume first that $f_{p, q}$ is isometric to $f$. By multiplying with a unit quaternion from the right we may assume that $p \in \mathbb{R}$. Then $\left|d f_{p, q}\right|=|d f|$ implies by (23)

$$
p^{2}+|q|^{2}+2 p \operatorname{Re}(R q)=|p+R q|^{2}=1
$$

and thus $\operatorname{Re}(R q)$ is constant. Since the stereographic projection of $R$ is a meromorphic function, the right normal $R$ can only take values in a plane if $R$ is constant. In other words, if $R$ is not constant then $\operatorname{Re}(R q)=\operatorname{Re}(R \operatorname{Im} q)=-<R, \operatorname{Im} q>$ is constant only if $\operatorname{Im} q=0$. But then the above equation gives $p^{2}+q^{2}=1$ with $q \in \mathbb{R}$ and $(p, q)=(\cos \theta, \sin \theta)$ for some $\theta \in \mathbb{R}$. If $R$ is constant then $d f^{*}=-* d f=d f R$ gives $f_{p, q}=f(p+R q)=f_{p+R q, 0}$ with constant $p+R q \in S^{3}$. (23).

Conversely, $f_{p, q}=f_{n \cos \theta, n \sin \theta}, n \in S^{3}$, then $|p+R q|=1$ and thus $\left|d f_{p, q}\right|=|d f|$ by

A similar argument shows the statement for the left associated family.

Remark 4.3 For any immersion $f: M \rightarrow \mathbb{R}^{3}=\operatorname{Im} \mathbb{H}$ in Euclidean 3-space, the left and right normal coincide. A surface in the right associated family $f_{p, q}$ of a minimal surface $f: M \rightarrow \mathbb{R}^{3}$ has left normal $N_{p, q}=N$ and right normal $R_{p, q}=(p+N q)^{-1} N(p+N q)$. In particular, we have $N_{p, q} \neq R_{p, q}$ in general and thus, elements of the right associated families of a minimal surface $f: M \rightarrow \mathbb{R}^{3}$ are not necessarily minimal in 3-space but are minimal surfaces in $\mathbb{R}^{4}$.

\subsection{The associated family of the harmonic conformal Gauss map}

We now give a derivation of the associated family of minimal surfaces in terms of the associated family of harmonic maps. Recall that in the case of a constant mean curvature surface $f: M \rightarrow \mathbb{R}^{3}$ the Gauss map $N: M \rightarrow S^{2}$ of $f$ is by the Ruh-Vilms theorem [63] harmonic and its associated family $\hat{d}_{\lambda}$ of flat connections (18) gives rise to the associated family of constant mean curvature surfaces: for $\mu \in \mathbb{C}_{*}$ the map $N$ is harmonic with respect to the quaternionic connection $\hat{d}_{\mu}$ for all $\mu \in S^{1}$, see e.g. [9] for details. Thus, for any $\hat{d}_{\mu^{-}}$ parallel section $\varphi \in \Gamma(\underline{\mathbb{H}})$ the map $N_{\mu}=\varphi^{-1} N \varphi$ is harmonic with respect to $d$. Note that $\varphi$ is unique up to a right multiplication by a constant quaternion. This family $N_{\lambda}, \lambda \in S^{1}$, is called the associated family of $N$. The Sym-Bobenko formula [10] relies on the link (4) between the differentials of $f$ and $N$ to obtain the constant mean curvature surface $f$ by differentiating a family $\varphi_{\lambda}$ of parallel sections of $d_{\lambda}$ with respect to the parameter $\lambda$. This way, one can obtain from the associated family $N_{\lambda}$ of $N$ a family of constant mean curvature surfaces, the associated family of $f$.

In the case of a minimal surface $f: M \rightarrow \mathbb{R}^{4}$ with right normal $R$, we have seen in Lemma 3.1 that every parallel section $\beta \in \Gamma(\underline{\mathbb{H}})$ of the associated family of flat connections of $R$ is, using (16), given by

$$
\beta=R m+m \frac{b}{a-1}, \quad m \in \mathbb{H}_{*},
$$

where $a=\frac{\mu+\mu^{-1}}{2}, b=i \frac{\mu^{-1}-\mu}{2}$, and $\mu \in \mathbb{C} \backslash\{0,1\}$.

If $\mu \in S^{1}, \mu \neq 1$, then $a=\operatorname{Re} \mu, b=\operatorname{Im} \mu \in \mathbb{R}$, and $\beta=\left(R+\frac{b}{a-1}\right) m$ with $m \in \mathbb{H}_{*}$. Thus, the associated family of harmonic maps is given by

$$
R_{\mu}=\beta^{-1} R \beta=m^{-1} R m .
$$


In particular, the harmonic map $R_{\mu}$ does not depend on the parameter $\mu$. In the case of a minimal surface, the equation (4) does not allow to reconstruct the minimal surface from the harmonic Gauss map. In particular, there is no Sym-Bobenko formula to obtain a minimal surface with right normal $R_{\mu}$ via differentiation of parallel sections with respect to the parameter $\mu$.

To obtain a non-trivial family of minimal surfaces with right normal $R_{\mu}$ for $\mu \in \mathbb{C}_{*}$, we consider instead the harmonic conformal Gauss map $S$ of $f$ :

Theorem 4.4 Let $f: M \rightarrow \mathbb{R}^{4}$ be a minimal surface with conformal Gauss map $S$. Then, up to Möbius transformation, the line bundle of a minimal surface $f_{\cos \theta, \sin \theta}, \theta \in \mathbb{R}$, in the associated family of $f$ is given by

$$
L_{\phi}=\phi^{-1} L
$$

where $\phi=\left(\varphi_{1}, \varphi_{2}\right)$ is an invertible endomorphism with $d_{\mu}$-parallel columns $\varphi_{1}, \varphi_{2}$, and $\mu=\cos (2 \theta)-i \sin (2 \theta) \in S^{1}$. Moreover,

$$
S_{\phi}=\phi^{-1} S \phi
$$

is the conformal Gauss map of $f_{\cos \theta}, \sin \theta$.

Proof For $\mu \in S^{1}$ and invertible $\phi$ and $\tilde{\phi}$ with $d_{\mu}$-parallel columns, we have $\tilde{\phi}=\phi B$ with $B$ constant so that $L_{\tilde{\phi}}=B^{-1} L_{\phi}$ is given by a Möbius transformation. Thus, we can assume without loss of generality that for $\mu \neq 1$

$$
\phi=(e, \varphi)
$$

where $\varphi=e \alpha+\psi \beta$ is a $d_{\mu}$-parallel section with nowhere vanishing $\beta$. Then

$$
\phi^{-1} \psi=\phi^{-1}(-e \alpha+\varphi) \beta^{-1}=\left(\begin{array}{c}
-\alpha \\
1
\end{array}\right) \beta^{-1}
$$

so that $L_{\phi}$ is the line bundle of the (branched) conformal immersion $f_{\phi}=-\alpha: M \rightarrow \mathbb{R}^{4}$. By Proposition 3.3 and (16) we have

$$
\alpha=-\left(f^{*}+f \frac{b}{a-1}\right) m
$$

since $\frac{b}{a-1} \in \mathbb{R}$ for $\mu \in S^{1}$. Writing $\mu=\cos \vartheta+i \sin \vartheta$ we see

$$
\frac{b}{a-1}=\frac{\sin \vartheta}{\cos \vartheta-1}=-\cot \frac{\vartheta}{2}
$$

We consider $\alpha$ up to Möbius transformations, and may thus assume without loss of generality that $m=-\sin \frac{\vartheta}{2}$ so that

$$
f_{\phi}=f_{\cos \theta, \sin \theta}
$$

for $\theta=-\frac{\vartheta}{2}$. For $\mu=1$ we can choose $\phi$ as the identity matrix, and obtain $f_{\phi}=f_{1,0}=f$.

By definition $S_{\phi}$ leaves $L_{\phi}$ invariant. The final statement follows by a similar argument as in [9]. In fact, this is a special case of a corresponding statement for (constrained) Willmore surfaces $f: M \rightarrow \mathbb{R}^{4}$, see $[4,8]$. 
We also get an interpretation of the right associated family: If we consider $\phi=(e, \varphi)$ with $d_{\mu}^{S} \varphi=0$ for some $\mu \in \mathbb{C} \backslash\{0,1\}$, then the line bundle $L_{\phi}=\phi^{-1} L$ gives by the same argument as above a member of the generalised associated family, up to Möbius transformation,

$$
f_{\phi}=\alpha(1-a)=f^{*} m(a-1)+f m b=f_{m b, m(a-1)} .
$$

Note however, that $\phi$ is not a $d_{\mu}^{S}$-parallel endomorphism since $d_{\mu}^{S}$ is a complex but not a quaternionic connection.

\section{Simple factor dressing}

As we have seen, the harmonic left and right normals and the harmonic conformal Gauss map of a minimal surface give rise to families of flat connections. Conversely, if a family of flat connections is of an appropriate form, it can be used to construct a harmonic map. In particular, if $d_{\lambda}$ is the associated family of flat connections of a harmonic map, one can gauge $d_{\lambda}$ with a $\lambda$-dependent dressing matrix $r_{\lambda}$ to obtain a new family of flat connections $\breve{d}_{\lambda}=r_{\lambda} \cdot d_{\lambda}$. If $r_{\lambda}$ satisfies appropriate reality and holomorphicity conditions, then $\breve{d}_{\lambda}$ is the associated family of a new harmonic map, a so-called dressing of the original harmonic map, see $[70,71]$.

\subsection{Simple factor dressing of the left and right normals}

We first consider the case when $R: M \rightarrow S^{2}$ is the harmonic right normal of a minimal surface in $\mathbb{R}^{4}$ and choose the simplest possible dressing matrix: If $d_{\lambda}$ is the associated family (12) of the harmonic map $R$ then the simple factor dressing matrix $r_{\lambda}$ is obtained by choosing $\mu \in \mathbb{C} \backslash\{0,1\}$ and a parallel section $\beta$ of the flat connection $d_{\mu}$ of the associated family as

$$
r_{\lambda} v= \begin{cases}v \frac{1-\bar{\mu}^{-1}}{1-\mu} \frac{\lambda-\mu}{\lambda-\bar{\mu}^{-1}}, & v \in \beta \mathbb{C} \\ v, & v \in(\beta \mathbb{C})^{\perp} .\end{cases}
$$

In [9] it is shown that $\breve{d}_{\lambda}=r_{\lambda} \cdot d_{\lambda}$ is the associated family of a harmonic map $\breve{R}: M \rightarrow S^{2}$, the simple factor dressing of $R$, and

$$
\breve{R}=\breve{T}^{-1} R \breve{T}
$$

where $\breve{T}=\frac{1}{2}\left(-R \beta(a-1) \beta^{-1}+\beta b \beta^{-1}\right)$ is given explicitly in terms of $\beta$ and $\mu$, where $a=\frac{\mu+\mu^{-1}}{2}, b=i \frac{\mu^{-1}-\mu}{2}$. In our case, any $d_{\mu}$-parallel section $\beta$ is given by Lemma 3.1 and (16) as

$$
\beta=(R+\rho) m, \quad m \in \mathbb{H}_{*}, \quad \rho=m \frac{b}{a-1} m^{-1} .
$$

Since $\beta$ is nowhere vanishing, we see that

$$
\breve{T}=\frac{1}{2}(-R \beta(a-1)+\beta b) \beta^{-1}=-(R+\rho)^{-1},
$$

and the simple factor dressing of $R$ is

$$
\breve{R}=(R+\rho) R(R+\rho)^{-1}, \quad \rho=m \frac{i(1+\mu)}{1-\mu} m^{-1}, \quad m \in \mathbb{H}_{*} .
$$


In particular, a simple factor dressing is determined by the choice $\mu \in \mathbb{C} \backslash\{0,1\}$, giving the pole of the simple factor, and $m \in \mathbb{H}_{*}$, giving the parallel bundle of $d_{\mu}$. A straight forward computation shows that

$$
d \breve{R}=\left(1+\rho^{2}\right)(R+\rho)^{-1} d R(R+\rho)^{-1} .
$$

Since

$$
\rho^{2}=(R+\rho-R)^{2}=(R+\rho)^{2}-R(R+\rho)-(R+\rho) R-1
$$

we see that

$$
(R+\rho)^{-1}\left(1+\rho^{2}\right)(R+\rho)^{-1}=1-(R+\rho)^{-1} R-R(R+\rho)^{-1} .
$$

The right hand side of this equation commutes with $R$ so that also

$$
\left[(R+\rho)^{-1}\left(1+\rho^{2}\right)(R+\rho)^{-1}, R\right]=0 .
$$

Therefore,

$$
(d \breve{R})^{\prime}=\frac{1}{2}(d \breve{R}-\breve{R} * d \breve{R})=\left(1+\rho^{2}\right)(R+\rho)^{-1} \frac{1}{2}(d R+R * d R)(R+\rho)^{-1}=0
$$

which shows that $\breve{R}$ is conformal and harmonic. Indeed, the simple factor dressing of the right normal of a minimal surface in $\mathbb{R}^{4}$ is the right normal of a minimal surface:

Theorem 5.1 Let $f: M \rightarrow \mathbb{R}^{4}$ be a minimal surface with right normal $R$.

Then every simple factor dressing of $R$ is the right normal $R_{p, q}$ of a minimal surface $f_{p, q}$ in the right associated family of $f$.

Proof Let $\mu \in \mathbb{C} \backslash\{0,1\}$ be the pole of the simple factor dressing and put, as usual, $a=$ $\frac{\mu+\mu^{-1}}{2}, b=\frac{i\left(\mu^{-1}-\mu\right)}{2}$. Write $\hat{c}=\mathrm{mcm}^{-1}$ for $c \in \mathbb{C}$ where $m \in \mathbb{H}_{*}$ determines the $d_{\mu^{-}}$ parallel bundle of the simple factor dressing.

Consider the minimal surface

$$
h=f \hat{b}+f^{*}(\hat{a}-1)
$$

in the associated family of $f$. From (24) we see that the right normal of $h=f_{\hat{b}, \hat{a}-1}$ is given by

$$
R_{h}=(\hat{a}-1)^{-1}(\rho+R)^{-1} R(\rho+R)(\hat{a}-1),
$$

where we used that $\hat{a} \neq 1$ since $\mu \neq 1$. Now, $\hat{a}^{2}+\hat{b}^{2}=1$ gives $1+\rho^{2}=1+\frac{\hat{b}^{2}}{(\hat{a}-1)^{2}}=-\frac{2}{\hat{a}-1}$. In particular, we have seen already (27) that $(R+\rho)^{-1} \frac{2}{\hat{a}-1}(R+\rho)^{-1}$ commutes with $R$ so that also

$$
[R,(R+\rho)(\hat{a}-1)(R+\rho)]=0 .
$$

Thus, the right normal

$$
R_{h}=(\rho+R) R(\rho+R)^{-1}
$$

of the associated minimal surface $h$ is (26) the dressing of $R$.

Remark 5.2 Note that the simple factor dressing of the harmonic right normal does not single out a canonical minimal surface with this right normal: the element $f_{\hat{b}, \hat{a}-1}$ is one example of such a minimal surface but so is $p f_{\hat{b}, \hat{a}-1}+q f_{\hat{b}, \hat{a}-1}^{*}$ for any $p, q \in \mathbb{H}_{*}$. 
An analogue theorem holds for the left normal $N$ of a minimal surface $f: M \rightarrow \mathbb{R}^{4}$ :

Theorem 5.3 The simple factor dressing of $N$ is the left normal $N^{p, q}$ of an element $f^{p, q}$ in the left associated family of $f$.

As noted before, if $f: M \rightarrow \mathbb{R}^{3}$ is a minimal surface in $\mathbb{R}^{3}$ then the left and right associated families give in general minimal surfaces in $\mathbb{R}^{4}$. In particular:

Corollary 5.4 Let $f: M \rightarrow \mathbb{R}^{3}$ be a minimal surface with Gauss map $N$ and assume that $f$ is not a plane. Let $f_{\hat{b}, \hat{a}-1}$ be the minimal surface in the associated family of $f$ whose right normal $R_{\hat{b}, \hat{a}-1}$ is the simple factor dressing of $N$ given by $\mu \in \mathbb{C} \backslash\{0,1\}, m \in \mathbb{H}_{*}$, where $\hat{a}=m \frac{\mu+\mu^{-1}}{2} m^{-1}, \hat{b}=m i \frac{\mu^{-1}-\mu}{2} m^{-1}$.

Then $f_{\hat{b}, \hat{a}-1}$ is a minimal surface in $\mathbb{R}^{3}$ if and only if $\mu \in S^{1}, \mu \neq 1$.

Proof If $f_{\hat{b}, \hat{a}-1}$ is in 3-space then $N_{\hat{b}, \hat{a}-1}=R_{\hat{b}, \hat{a}-1}$, that is by (29)

$$
N=(\rho+N) N(\rho+N)^{-1}
$$

with $\rho=\frac{\hat{b}}{\hat{a}-1}$. But then $[\rho, N]=0$ and, since $\rho$ is constant, we see that $\rho=m i \frac{1+\mu}{1-\mu} m^{-1} \in \mathbb{R}$. In particular, $i \frac{1+\mu}{1-\mu} \in \mathbb{R}$ which is equivalent to $\mu \in S^{1}, \mu \neq 1$.

Conversely, for $\mu \in S^{1}$ we know $\hat{a}, \hat{b} \in \mathbb{R}$ so that $f_{\hat{b}, \hat{a}-1}=f \hat{b}+f^{*}(\hat{a}-1)$ takes values in $\mathbb{R}^{3}$.

We will use Remark 5.2 to obtain a minimal surface in 3-space with a given simple factor dressing as its Gauss map. This operation turns out to be the surface obtained by applying a corresponding simple factor dressing on the conformal Gauss map.

\subsection{Simple factor dressing of a minimal surface}

The conformal Gauss map of a Willmore surface is harmonic and one can define a dressing on it $[4,61]$. Since the conformal Gauss map determines a conformal immersion (if the Hopf field is not zero), this induces a transformation, a dressing, on Willmore surfaces. (Actually, Burstall and Quintino define more generally a dressing on constrained Willmore surfaces).

We will again only discuss the special case of simple factor dressing by choosing the simplest possible dressing matrix. As before, the simple factor dressing of the conformal Gauss map $S$ of a Willmore surface $f: M \rightarrow S^{4}$ is given explicitly by parallel sections of a connection $d_{\mu}^{S}$ of the associated family of flat connections [43]: for $\mu \in \mathbb{C} \backslash\{0,1\}$ let $W_{\mu}$ be a $d_{\mu}^{S}$-stable, complex rank 2 bundle over $\tilde{M}$ with $W_{\mu} \oplus W_{\mu} j=\underline{\widetilde{\mathbb{H}}}^{2}$. For two $d_{\mu}^{S}$-parallel sections $\varphi_{1}, \varphi_{2} \in \Gamma\left(W_{\mu}\right)$ with $\phi=\left(\varphi_{1}, \varphi_{2}\right)$ regular, define a conformal immersion $\hat{f}: \tilde{M} \rightarrow S^{4}$ by

$$
\hat{L}=\left(S+\phi \frac{b}{a-1} \phi^{-1}\right) L,
$$

where $\frac{b}{a-1}$ is the left multiplication by the quaternion $\frac{b}{a-1}$ on $\underline{\mathbb{H}}^{2}$ and as usual $a=\frac{\mu+\mu^{-1}}{2}, b=$ $i \frac{\mu^{-1}-\mu}{2}$. Then the conformal Gauss map $\hat{S}$ of $\hat{f}$ is the simple factor dressing of $S$. In particular, $\hat{S}$ is harmonic, and $\hat{f}$ is a Willmore surface. It is known that $\hat{L}$, and thus $\hat{f}$, is independent of the choice of basis for $W_{\mu}$ [43]. We call the Willmore surface $\hat{f}$ a simple factor dressing of $f$. Note that $\frac{b}{a-1} \in \mathbb{R}$ for $\mu \in S^{1}$ so that $\hat{f}=f$ in this case. 
Note that this gives a conformal theory. However, in the case of a minimal surface $f$ : $M \rightarrow \mathbb{R}^{4}$ we are only interested in the Euclidean theory, that is, simple factor dressings which are again surfaces in the same 4-space. Thus, we will restrict to simple factor dressings such that $S+\phi \frac{b}{a-1} \phi^{-1}$ stabilises the point at infinity $\infty=e \mathbb{H}$. Because $S e=e N$ by (9) where $N$ is the left normal of $f$, we have to restrict to $\phi$ with $\phi \frac{b}{a-1} \phi^{-1} \infty=\infty$. Since $\frac{b}{a-1} \in \mathbb{R}$ if and only if $\mu \in S^{1}$ we can assume that $\frac{b}{a-1}$ is not real as otherwise $\hat{f}=f$.

We recall that any $d_{\mu}^{S}$-parallel section $\varphi \neq e n, n \in \mathbb{H}$, is given by Proposition 3.3 and (16) as $\varphi=e \alpha+\psi \beta$ where $\alpha=-\left(f^{*}+f \rho\right) m, \beta=(R+\rho) m$ with $\rho=m \frac{i(1+\mu)}{1-\mu} m^{-1}$ and $m \in \mathbb{H}_{*}$. In particular, for regular $\phi=\left(\varphi_{1}, \varphi_{2}\right)$ with two such $d_{\mu}^{S}$-parallel sections $\varphi_{1}, \varphi_{2}$ we write

$$
\phi=\left(\begin{array}{ll}
\alpha_{1} & \alpha_{2} \\
\beta_{1} & \beta_{2}
\end{array}\right), \quad \phi^{-1}=\left(\begin{array}{ll}
\zeta_{1} & \xi_{1} \\
\zeta_{2} & \xi_{2}
\end{array}\right)
$$

in the basis $(e, \psi)$ where

$$
\alpha_{1}=f_{-m_{1} \frac{b}{a-1},-m_{1}}, \quad \alpha_{2}=f_{-m_{2} \frac{b}{a-1},-m_{2}}, \quad m_{1}, m_{2} \in \mathbb{H}_{*},
$$

are in the right associated family of $f$, and

$$
\beta_{1}=R m_{1}+m_{1} \frac{b}{a-1}, \quad \beta_{2}=R m_{2}+m_{2} \frac{b}{a-1}
$$

are nowhere vanishing parallel sections with respect to the connection $d_{\mu}$ of the associated family of connections of the right normal $R$. Assume that $\phi \frac{b}{a-1} \phi^{-1}$ fixes the point at infinity. Then there exists $\eta: M \rightarrow \mathbb{H}_{*}$ with

$$
\frac{b}{a-1}\left(\begin{array}{l}
\zeta_{1} \\
\zeta_{2}
\end{array}\right)=\left(\begin{array}{l}
\zeta_{1} \\
\zeta_{2}
\end{array}\right) \eta .
$$

Since $\beta_{1}, \beta_{2}$ are nowhere vanishing so is $\zeta_{1}$ and the equation above shows that $\zeta_{2} \zeta_{1}^{-1}$ commutes with the complex number $\frac{b}{a-1} \notin \mathbb{R}$. Therefore, we have $\zeta_{2}=q \zeta_{1}$ with $q: M \rightarrow \mathbb{C}_{*}$ and, because $\phi \phi^{-1}=$ Id and $\zeta_{1}$ is nowhere vanishing, we conclude $\beta_{1}+\beta_{2} q=0$.

Both $\beta_{1}$ and $\beta_{2}$ are $d_{\mu}$-parallel, and thus, $q \in \mathbb{C}_{*}$ is constant.

If $R$ is constant, that is, if $f$ is the twistor projection of a holomorphic curve in $\mathbb{C P}^{3}$, then $f^{*}=f R+c$ with some $c \in \mathbb{H}$, so that with (30) and (31) we obtain $\varphi_{1}+\varphi_{2} q=e n$ with $n=-c\left(m_{2} q+m_{1}\right)$ and $q \in \mathbb{C}_{*}$. In other words, en is a $d_{\mu}^{S}$-parallel section of $W_{\mu}$ and we can replace $\phi$ in the definition of $\hat{S}$ by $\tilde{\phi}=\left(e n, \varphi_{2}\right)$.

If $R$ is not constant, we use again the explicit forms (31) to obtain from $\beta_{1}+\beta_{2} q=0$, $q \in \mathbb{C}_{*}$, that $R\left(m_{1}+m_{2} q\right)$ is constant, which implies that $m_{1}=-m_{2} q$. But then (30) and (31) show that $\varphi_{1}=-\varphi_{2} q$ which contradicts the assumption that $\phi$ is regular.

Thus, from now on we can restrict to regular endomorphism $\phi$ of the form

$$
\phi=\left(\begin{array}{ll}
n & \alpha \\
0 & \beta
\end{array}\right)
$$

in the basis $(e, \psi)$ where $n \in \mathbb{H}_{*}$ and $\varphi=e \alpha+\psi \beta, \beta$ nowhere vanishing, is a parallel section of $d_{\mu}^{S}$ to obtain all simple factor dressings in 4-space.

Theorem 5.5 Let $f: M \rightarrow \mathbb{R}^{4}$ be a minimal surface and $\hat{f}: \tilde{M} \rightarrow \mathbb{R}^{4}$ a simple factor dressing of $f$ in 4-space. 
Then $\hat{f}$ is a minimal surface, and $\hat{f}$ is preserved when changing the parameters $(\mu, m, n)$ to $(\mu, m z, n w)$ or $\left(\bar{\mu}^{-1}, m j, n j\right)$ for $z, w \in \mathbb{C}_{*}$. For $\mu \in S^{1}$ the simple factor dressing $\hat{f}=f$ is trivial.

Proof Since $\psi \beta \in \Gamma(L)$ is nowhere vanishing, the line bundle $\hat{L}$ is spanned by

$$
\hat{\varphi}=\left(S+\phi \frac{b}{a-1} \phi^{-1}\right)(\varphi-e \alpha) .
$$

Since $S e=e N$ and $S \varphi=e N \alpha-\psi R \beta$ by (9) we conclude

$$
\hat{\varphi}=\left(\begin{array}{c}
N \alpha \\
-R \beta
\end{array}\right)+\left(\begin{array}{c}
-N \alpha \\
0
\end{array}\right)+\left(\begin{array}{c}
\alpha \frac{b}{a-1} \\
\beta \frac{b}{a-1}
\end{array}\right)+\left(\begin{array}{c}
-n \frac{b}{a-1} n^{-1} \alpha \\
0
\end{array}\right)=\left(\begin{array}{c}
\alpha \frac{b}{a-1}-n \frac{b}{a-1} n^{-1} \alpha \\
-R \beta+\beta \frac{b}{a-1}
\end{array}\right)
$$

in the basis $(e, \psi)$. Recalling (14), that is, $-R \beta+\beta \frac{b}{a-1}=-2 m(a-1)^{-1}$, we see that

$$
\hat{f}=f-\left(\alpha \frac{b}{a-1}-n \frac{b}{a-1} n^{-1} \alpha\right) \frac{a-1}{2} m^{-1} .
$$

We substitute $\alpha=-f m \frac{b}{a-1}-f^{*} m$ and use $\hat{a}^{2}+\hat{b}^{2}=1$ for $\hat{a}=\mathrm{mam}^{-1}, b=m b m^{-1}$, and $\tilde{\rho}=n \frac{b}{a-1} n^{-1}$, to obtain

$$
\hat{f}=\frac{1}{2}\left(-f(\hat{a}-1)+f^{*} \hat{b}-\tilde{\rho}\left(f \hat{b}+f^{*}(\hat{a}-1)\right)\right) .
$$

Thus,

$$
\hat{f}=-\frac{\tilde{\rho}}{2} h+\frac{1}{2} h^{*}=h^{-\frac{\tilde{\rho}}{2}, \frac{1}{2}}
$$

is an element of the left associated family of the minimal surface

$$
h=f \hat{b}+f^{*}(\hat{a}-1)=f_{\hat{b},(\hat{a}-1)}
$$

in the right associated family of $f$. In particular, $\hat{f}$ is minimal. From the explicit formula above we see that $\hat{f}$ is preserved when changing $(\mu, m, n)$ to $(\mu, m z, n w)$ with $z, w \in \mathbb{C}_{*}$. Since $\frac{\bar{\mu}^{-1}+\bar{\mu}}{2}=\bar{a}, i \frac{\bar{\mu}-\bar{\mu}^{-1}}{2}=\bar{b}$ and $(m j) \bar{z}(m j)^{-1}=m z m^{-1}$ for all $z \in \mathbb{C}, m \in \mathbb{H}_{*}$, we obtain the same simple factor dressing for the parameters $(\mu, m, n)$ and $\left(\bar{\mu}^{-1}, m j, n j\right)$. The final statement follows from the fact that $\frac{b}{a-1} \in \mathbb{R}$ for $\mu \in S^{1}$.

Remark 5.6 Note that the last statements in the above corollary are special cases of more general facts for simple factor dressings of Willmore surfaces: since the simple factor dressing is independent of the choice of basis of $W_{\mu}$ and the family of flat connections satisfies a reality condition [9], the surface is preserved under the given changes of parameter. The last statement holds for general simple factor dressings with $\mu \in S^{1}$.

In particular, we emphasise again that in contrast to the simple factor dressing of the right and left normal, the simple factor dressing of the conformal Gauss map associates a unique minimal surface:

Definition 5.7 The simple factor dressing of a minimal surface $f: M \rightarrow \mathbb{R}^{4}$ with parameters $(\mu, m, n)$ is the minimal surface $\hat{f}: \tilde{M} \rightarrow \mathbb{R}^{4}$ given by 


$$
\hat{f}=-f \frac{m(a-1) m^{-1}}{2}+f^{*} \frac{m b m^{-1}}{2}-n \frac{b}{a-1} n^{-1}\left(f \frac{m b m^{-1}}{2}+f^{*} \frac{m(a-1) m^{-1}}{2}\right)
$$

where $m, n \in S^{3}, \mu \in \mathbb{C} \backslash\{0,1\}$ and $a=\frac{\mu+\mu^{-1}}{2}, b=i \frac{\mu^{-1}-\mu}{2}$.

If $m=n=1$ then we refer to $f^{\mu}=\hat{f}$ as the simple factor dressing of $f$ with parameter $\mu$.

The simple factor dressing with parameter $\mu$ of the rigid motion $\tilde{f}=n^{-1} f m$ of $f$ is given by

$$
\tilde{f}^{\mu}=-n^{-1} f m \frac{a-1}{2}+n^{-1} f^{*} m \frac{b}{2}-\frac{b}{a-1}\left(n^{-1} f m \frac{b}{2}+n^{-1} f^{*} m \frac{a-1}{2}\right)=n^{-1} \hat{f} m,
$$

where $\hat{f}$ is the simple factor dressing (32) of $f$ with parameters $(\mu, m, n)$. Thus, all simple factor dressings are build from rigid motions of the simple factor dressings with parameter $\mu$ :

Proposition 5.8 Let $\hat{f}$ be a simple factor dressing of a minimal surface $f: M \rightarrow \mathbb{R}^{4}$ with parameters $(\mu, m, n)$. Then

$$
\hat{f}=\mathcal{R}_{n, m}\left(\left(\mathcal{R}_{n, m}^{-1}(f)\right)^{\mu}\right)
$$

where $\left(\mathcal{R}_{n, m}^{-1}(f)\right)^{\mu}$ is the simple factor dressing of the rotated surface $\mathcal{R}_{n, m}^{-1}(f)=n^{-1} f m$ with parameter $\mu$.

Since the associated families of the left and right normals and the conformal Gauss maps are related, we also have a correspondence between the resulting simple factor dressings:

Corollary 5.9 The simple factor dressing of a minimal immersion $f: M \rightarrow \mathbb{R}^{4}$ with parameters $(\mu, m, n)$ is a minimal immersion $\hat{f}: \tilde{M} \rightarrow \mathbb{R}^{4}$.

The right and left normal of $\hat{f}$ are given by simple factor dressings of the right and left normal of $f$ respectively. Moreover, $\hat{f}$ is complete if and only if $f$ is complete.

Proof The differential of the simple factor dressing $\hat{f}$ with parameters $(\mu, m, n)$ is given by

$$
d \hat{f}=-\left(N+n \frac{b}{a-1} n^{-1}\right) \frac{d f}{2}\left(R+m \frac{b}{a-1} m^{-1}\right) m(a-1) m^{-1}
$$

where we used that $d f^{*}=-* d f$ and $* d f=N d f=-d f R$. In particular, the right normal

$$
\hat{R}=m(a-1)^{-1} m^{-1}\left(R+m \frac{b}{a-1} m^{-1}\right)^{-1} R\left(R+m \frac{b}{a-1} m^{-1}\right) m(a-1) m^{-1}
$$

and left normal

$$
\hat{N}=\left(N+n \frac{b}{a-1} n^{-1}\right) N\left(N+n \frac{b}{a-1} n^{-1}\right)^{-1}
$$

of $\hat{f}$ are by (28) and (29) the simple factor dressings of the right and left normal of $f$ which are given by the pole $\mu$ and the parallel sections $R m+m \frac{b}{a-1}$ and $N n+n \frac{b}{a-1}$ respectively. 
Finally, $\hat{f}$ is branched at $p$ if and only if $\left(N(p)+n \frac{b}{a-1} n^{-1}\right)=0$ or $\left(R(p)+m \frac{b}{a-1} m^{-1}\right)=$ 0 . We already have seen that $\beta=R m+m \frac{b}{a-1}$ is nowhere vanishing if $m \neq 0$. A similar argument, as given before Lemma 3.1, gives the corresponding statement for the expression in $N$, so that $f$ and $\hat{f}$ have the same conformal class, that is,

$$
|d \hat{f}|=r|d f|
$$

with $r: M \rightarrow(0, \infty)$. In particular, the simple factor dressing $\hat{f}$ of a minimal immersion has no branch points, and $\hat{f}$ is complete if and only if $f$ is complete.

From the explicit form (32) of the simple factor dressing of a minimal surface we immediately see that the simple factor dressing commutes with the conjugation:

Corollary 5.10 Let $f: M \rightarrow \mathbb{R}^{4}$ be a minimal surface and $f^{*}$ a conjugate surface of $f$. Then a conjugate surface of the simple factor dressing of $f$ is given by a simple factor dressing of the conjugate surface $f^{*}$.

Moreover, the choice of a different conjugate surface results in a translation of the simple factor dressing in 4-space.

\section{Simple factor dressing and the López-Ros deformation}

Given a minimal surface $f: M \rightarrow \mathbb{R}^{4}$ in 4-space with Weierstrass data $\left(g_{1}, g_{2}, \omega\right)$ denote, in analogy to the case of a minimal surface in $\mathbb{R}^{3}$, by $f^{\sigma}$ the López-Ros deformation of $f$ with complex parameter $\sigma \in \mathfrak{C}$, that is, the minimal surface given by the Weierstrass data $\left(\sigma g_{1}, \sigma g_{2}, \frac{\omega}{\sigma}\right)$. Similarly, the Goursat transformation is defined by $\operatorname{Re}\left(\mathcal{A}\left(f+\mathbf{i} f^{*}\right)\right)$ where $\mathcal{A} \in \mathrm{O}(4, \mathfrak{C})$ and $f^{*}$ is a conjugate surface of $f$. In this section, we will show that the López-Ros deformation is a special case of the simple factor dressing. Indeed, all simple factor dressings are (special) Goursat transformations.

\subsection{The López-Ros deformation in $\mathbb{R}^{4}$}

Since by Proposition 5.8 any simple factor dressing is given in terms of the simple factor dressing with parameter $\mu$, we will first show that these simple factor dressings are Goursat transformations.

Theorem 6.1 Let $f: M \rightarrow \mathbb{R}^{4}$ be a minimal surface in $\mathbb{R}^{4}$. Then the simple factor dressing with parameter $\mu$ of $f=f_{0}+f_{1} i+f_{2} j+f_{3} k$ is given by

$$
f^{\mu}=\left(\begin{array}{c}
f_{0} \\
f_{1} \\
\cos t\left(f_{2} \cosh s-f_{3}^{*} \sinh s\right)-\sin t\left(f_{3} \cosh s+f_{2}^{*} \sinh s\right) \\
\sin t\left(f_{2} \cosh s-f_{3}^{*} \sinh s\right)+\cos t\left(f_{3} \cosh s+f_{2}^{*} \sinh s\right)
\end{array}\right)
$$

where $s=-\ln |\mu|, t=\arg \frac{\bar{\mu}-1}{\bar{\mu}(1-\mu)}$. In particular, $f^{\mu}$ is a Goursat transform of $f$ whose holomorphic null curve is

$$
\Phi^{\mu}=\mathcal{L}^{\mu} \Phi
$$


where $\Phi$ is the holomorphic null curve of $f$ and

$$
\mathcal{L}^{\mu}=\left(\begin{array}{cccc}
1 & 0 & 0 & 0 \\
0 & 1 & 0 & 0 \\
0 & 0 & \cosh w & \mathbf{i} \sinh w \\
0 & 0 & -\mathbf{i} \sinh w & \cosh w
\end{array}\right) \in \mathrm{O}(4, \mathfrak{C})
$$

with $w=s+\mathbf{i} t$.

Proof Let $\mu \in \mathbb{C} \backslash\{0,1\}$ and put, as usual, $a=\frac{\mu+\mu^{-1}}{2}, b=i \frac{\mu^{-1}-\mu}{2}$. The simple factor dressing of $f$ with parameter $\mu$ is given by

$$
f^{\mu}=-f \frac{a-1}{2}+f^{*} \frac{b}{2}-\frac{b}{a-1}\left(f \frac{b}{2}+f^{*} \frac{a-1}{2}\right)=T_{1}(f)+T_{2}\left(f^{*}\right),
$$

where

$$
T_{1}(v)=-v \frac{a-1}{2}-\frac{b}{a-1} v \frac{b}{2} \quad \text { and } \quad T_{2}(v)=v \frac{b}{2}-\frac{b}{a-1} v \frac{a-1}{2}, \quad v \in \mathbb{H} .
$$

Next, we observe for $v \in \mathbb{C}=\operatorname{span}_{\mathbb{R}}\{1, i\}$ that

$T_{1}(v)=-\frac{1}{2}\left(v(a-1)+\frac{b}{a-1} v b\right)=v \quad$ and $\quad T_{2}(v)=\frac{1}{2}\left(v b-\frac{b}{a-1} v(a-1)\right)=0$,

where we used that $a^{2}+b^{2}=1$. To compute $T_{1}(v), T_{2}(v)$ for $v \in \mathbb{C} j=\operatorname{span}\{j, k\}$ we recall that

$$
\frac{\bar{\mu}-1}{\bar{\mu}(1-\mu)}=e^{s+i t}
$$

by definition of $s$ and $t$. Thus, with $a-1=\frac{(1-\mu)^{2}}{2 \mu}, b=i \frac{1-\mu^{2}}{2 \mu}$ we have

$$
\frac{|a-1|^{2}+|b|^{2}}{2|a-1|}=\cosh s, \quad \frac{\operatorname{Im}(b(\bar{a}-1))}{|a-1|}=\sinh s, \quad \text { and } \quad \frac{a-1}{|a-1|}=-e^{-i t} .
$$

Therefore, since $w v=v \bar{w}$ for every $w \in \mathbb{C}$ and $v \in \mathbb{C} j$, we see

$$
T_{1}(v)=-\frac{1}{2}\left(v(a-1)+\frac{b}{a-1} v b\right)=v e^{-i t} \cosh s
$$

and

$$
T_{2}(v)=\frac{1}{2}\left(v b-\frac{b}{a-1} v(a-1)\right)=-v i e^{-i t} \sinh s .
$$

Decomposing $f=\left(f_{0}+f_{1} i\right)+\left(f_{2} j+f_{3} k\right)$ and $f^{*}=\left(f_{0}^{*}+f_{1}^{*} i\right)+\left(f_{2}^{*} j+f_{3}^{*} k\right)$, the simple factor dressing of $f$ with parameter $\mu$ is then given by

$f^{\mu}=T_{1}(f)+T_{2}\left(f^{*}\right)=\left(f_{0}+f_{1} i\right)+\left(f_{2} j+f_{3} k\right) e^{-i t} \cosh s-\left(f_{2}^{*} j+f_{3}^{*} k\right) i e^{-i t} \sinh s$

which gives (34). The final statement follows by a straight forward computation of the holomorphic null curve.

By Proposition 5.8 we immediately see that the general simple factor dressing is a Goursat transformation, too.

Theorem 6.2 The simple factor dressing of a minimal surface $f: M \rightarrow \mathbb{R}^{4}$ is a Goursat transformation of $f$. 
Proof Let $\mu \in \mathbb{C} \backslash\{0,1\}$, then by Proposition 5.8 the simple factor dressing $\hat{f}$ with parameters $(\mu, m, n)$ is given by

$$
\hat{f}=\mathcal{R}_{n, m}\left(\left(\mathcal{R}_{n, m}^{-1}(f)\right)^{\mu}\right)
$$

where $\mathcal{R}_{n, m} \in \operatorname{SO}(4, \mathbb{R})$ is the map $v \mapsto n v m^{-1}$ and $\left(\mathcal{R}_{n, m}^{-1}(f)\right)^{\mu}$ is the simple factor dressing of $\tilde{f}=n^{-1} \mathrm{fm}$ with parameter $\mu$. If $\Phi$ denotes the holomorphic null curve of $f$ then the null curve of $\mathcal{R}_{n, m}^{-1}(f)$ is $\mathcal{R}_{n, m}^{-1} \Phi$ since $\mathcal{R}_{n, m}$ is real. But then the holomorphic null curve of the simple factor dressing of $\mathcal{R}_{n, m}^{-1}(f)$ with parameter $\mu$ is $\mathcal{L}^{\mu} \mathcal{R}_{n, m}^{-1} \Phi$ by (35). Thus, the holomorphic null curve of the simple factor dressing $\hat{f}$ with parameters $(\mu, m, n)$ is given by

$$
\hat{\Phi}=\mathcal{R}_{n, m} \mathcal{L}^{\mu} \mathcal{R}_{n, m}^{-1} \Phi
$$

But $\mathcal{R}_{n, m} \mathcal{L}^{\mu} \mathcal{R}_{n, m}^{-1} \in \mathrm{O}(4, \mathfrak{C})$ so that $\hat{f}$ is a Goursat transformation of $f$.

Note that the simple factor dressing is a special case of the Goursat transformation: its matrix $\mathcal{A} \in \mathrm{O}(4, \mathfrak{C})$ has $\operatorname{det} \mathcal{A}=1$ and special behaviour of the eigenspaces.

As before the López-Ros deformation can be given in terms of the surface and its conjugate which immediately shows that it is a special case of the simple factor dressing:

Theorem 6.3 Let $f: M \rightarrow \mathbb{R}^{4}$ be a minimal surface in $\mathbb{R}^{4}$ with conjugate surface $f^{*}$ and let $\sigma=e^{s+\mathbf{i} t} \in \mathfrak{C}_{*}$. Then the López-Ros deformation $f^{\sigma}$ of $f$ is given by

$$
f^{\sigma}=\left(\begin{array}{c}
f_{0} \\
\cos t\left(f_{1} \cosh s-f_{2}^{*} \sinh s\right)-\sin t\left(f_{2} \cosh s+f_{1}^{*} \sinh s\right) \\
\sin t\left(f_{1} \cosh s-f_{2}^{*} \sinh s\right)+\cos t\left(f_{2} \cosh s+f_{1}^{*} \sinh s\right) \\
f_{3}
\end{array}\right)
$$

where $f_{l}$ and $f_{l}^{*}$ are the coordinates of $f=f_{0}+f_{1} i+f_{2} j+f_{3} k$ and $f^{*}=f_{0}^{*}+f_{1}^{*} i+$ $f_{2}^{*} j+f_{3}^{*} k$ respectively.

In particular, the López-Ros deformation $f^{\sigma}$ of $f$ with parameter $\sigma=e^{s+\mathbf{i} t} \in \mathfrak{C}_{*},|\sigma| \neq$ 1 , is the simple factor dressing $\hat{f}$ of $f$ with parameters $(\mu, m, m)$ where $\mu=\frac{1-e^{-(s+i t)}}{1-e^{s-i t}} \in$ $\mathbb{C} \backslash\{0,1\}$ and $m=\frac{1-i-j-k}{2} \in S^{3}$.

From this we see again that the López-Ros deformation is a trivial rotation in the $i j$-plane if $\sigma \in S^{1} \subset \mathfrak{C}$. Moreover, if $\sigma \in \mathbb{R}$ then $\mu=-\frac{1}{\sigma}$.

Proof Let $f^{\sigma}$ be the Lopez-Ros deformation of $f$ with parameter $\sigma=e^{s+\mathbf{i} t} \in \mathfrak{C}_{*},|\sigma| \neq 1$. The first equation (36) is an analogue computation as in the proof of Theorem 2.4.

By assumption $|\sigma| \neq 1$ so that $\mu=\frac{1-e^{-(s+i t)}}{1-e^{s-i t}} \in \mathbb{C} \backslash\{0,1\}$ is well defined. Put, as usual, $a=\frac{\mu+\mu^{-1}}{2}, b=i \frac{\mu^{-1}-\mu}{2}$.

By Proposition 5.8, the simple factor dressing with parameters $(\mu, m, m)$ is $\hat{f}=$ $\mathcal{R}_{m, m}\left(\left(\mathcal{R}_{m, m}^{-1}(f)\right)^{\mu}\right)$ where $\left(\mathcal{R}_{m, m}^{-1}(f)\right)^{\mu}$ is the simple factor dressing of $\tilde{f}=\mathcal{R}_{m, m}^{-1}(f)=$ $m^{-1} f m$ with parameter $\mu$. Decomposing $f=\left(f_{0}+f_{1} i\right)+\left(f_{2} j+f_{3} k\right)$ we have $\tilde{f}=\left(f_{0}+f_{3} i\right)+\left(f_{1} j+f_{2} k\right)$ for $m=\frac{1-i-j-k}{2}$, and the simple factor dressing of $\tilde{f}$ with parameter $\mu$ is given by Theorem 6.1 as

$$
\tilde{f}^{\mu}=\left(f_{0}+f_{3} i\right)+\left(f_{1} j+f_{2} k\right) e^{-i t} \cosh s-\left(f_{1}^{*} j+f_{2}^{*} k\right) i e^{-i t} \sinh s .
$$


Therefore, the simple factor dressing of $f$ with parameters $(\mu, m, m)$

$$
\hat{f}=\mathcal{R}_{m, m} \tilde{f}^{\mu}=f_{0}+f_{3} k+\left(f_{1} i+f_{2} j\right) e^{-k t} \cosh s-\left(f_{1}^{*} i+f_{2}^{*} j\right) k e^{-k t} \sinh s=f^{\sigma}
$$

is indeed by (36) the López-Ros deformation of $f$.

Remark 6.4 In particular, with Proposition 5.8 we see that all simple factor dressings of a minimal surface are given, up to rotations, by the López-Ros deformation applied to a rigid motion of $f$.

If $f: M \rightarrow \mathbb{R}^{4}$ is a periodic minimal surface then the periods of the simple factor dressing $f^{\mu}$ with parameter $\mu$ are immediately given by the explicit formulation (34):

Corollary 6.5 If $f: M \rightarrow \mathbb{R}^{4}$ is a periodic minimal surface with translational periods $\gamma^{*} f=f+\tau$ for $\gamma \in \pi_{1}(M), \tau=\left(\tau_{0}, \tau_{1}, \tau_{2}, \tau_{3}\right)$, then the simple factor dressing $f^{\mu}$ of $f$ with parameter $\mu$ is periodic with $\gamma^{*} f^{\mu}=f^{\mu}+\tau^{\mu}$ where

$$
\tau^{\mu}=\left(\begin{array}{c}
\tau_{0} \\
\tau_{1} \\
\cos t\left(\tau_{2} \cosh s-\tau_{3}^{*} \sinh s\right)-\sin t\left(\tau_{3} \cosh s+\tau_{2}^{*} \sinh s\right) \\
\sin t\left(\tau_{2} \cosh s-\tau_{3}^{*} \sinh s\right)+\cos t\left(\tau_{3} \cosh s+\tau_{2}^{*} \sinh s\right)
\end{array}\right) .
$$

Here $\tau^{*}=\left(\tau_{0}^{*}, \tau_{1}^{*}, \tau_{2}^{*}, \tau_{3}^{*}\right)$ denote the periods of a conjugate surface $f^{*}$ of $f$, that is, $\gamma^{*} f^{*}=f^{*}+\tau^{*}$, and $s=-\ln |\mu|, t=\arg \frac{\bar{\mu}-1}{\bar{\mu}(1-\mu)}$.

In particular, $f^{\mu}$ is closed along $\gamma \in \pi_{1}(M)$, that is, $\gamma^{*} f^{\mu}=f^{\mu}$, if and only if

$$
\tau_{0}=\tau_{1}=0 \text { and }\left(\begin{array}{c}
\tau_{2} \\
\tau_{3}
\end{array}\right)=\left(\begin{array}{c}
\tau_{3}^{*} \\
-\tau_{2}^{*}
\end{array}\right) \tanh s .
$$

From this, we can immediately compute the periods of all simple factor dressings by Proposition 5.8.

In particular, assume that $f: M \rightarrow \mathbb{R}^{4}$ is single-valued on $M$, and that there exist $m, n \in \mathbb{H}_{*}$ such that all periods of the conjugate surface $f^{*}$ can be rotated simultaneously into the $1, i$-plane, that is,

$$
\mathcal{R}_{n, m}^{-1} \tau_{\gamma}^{*} \in \operatorname{span}\{1, i\}
$$

for all $\gamma \in \pi_{1}(M)$ where $\gamma^{*} f^{*}=f^{*}+\tau_{\gamma}^{*}$. Then all minimal surfaces in the complex 1-parameter family given by the simple factor dressings with parameters $(\mu, m, n), \mu \in$ $\mathbb{C} \backslash\{0,1\}$, are single-valued on $M$.

Finally, since a simple factor dressing of a finite total curvature minimal surface is given by a Goursat transformation, it has again finite total curvature:

Theorem 6.6 If $f: M \rightarrow \mathbb{R}^{4}$ has finite total curvature and if the simple factor dressing $\hat{f}: M \rightarrow \mathbb{R}^{4}$ of $f$ with parameters $(\mu, m, n)$ is single-valued on $M$ then $\hat{f}$ has finite total curvature.

Proof Since $f$ has finite total curvature, we can assume by Theorem 2.1 that $M=$ $\bar{M} \backslash\left\{p_{1}, \ldots, p_{r}\right\}$ where $\bar{M}$ is a Riemann surface punctured at finitely many $p_{i}$. Moreover, if $\Phi$ denotes the holomorphic null curve of $f$ then we can assume that $d \Phi$ extends meromorphically into the $p_{i}$. Since the simple factor dressing is a Goursat transformation, the holomorphic null curve $\hat{\Phi}$ of $\hat{f}$ is given by $\hat{\Phi}=\mathcal{A} \Phi$ with $\mathcal{A} \in \mathrm{O}(4, \mathfrak{C})$. Thus, $d \hat{\Phi}$ extends meromorphically into the punctures $p_{i}$. 


\subsection{Simple factor dressing in $\mathbb{R}^{3}$}

Given a minimal surface in $\mathbb{R}^{3}$ we now discuss when the simple factor dressing $\hat{f}$ is a minimal surface in 3-space. Considering a surface in $\mathbb{R}^{3}=\operatorname{Im} \mathbb{H}$ as a surface in $\mathbb{H}$ with vanishing real part, we immediately see with Theorem 6.1 that a simple factor dressing of $f$ with parameter $\mu \in \mathbb{C} \backslash\{0,1\}$ gives a minimal surface

$$
f^{\mu}=\left(\begin{array}{c}
f_{1} \\
\cos t\left(f_{2} \cosh s-f_{3}^{*} \sinh s\right)-\sin t\left(f_{3} \cosh s+f_{2}^{*} \sinh s\right) \\
\sin t\left(f_{2} \cosh s-f_{3}^{*} \sinh s\right)+\cos t\left(f_{3} \cosh s+f_{2}^{*} \sinh s\right)
\end{array}\right)
$$

in $\mathbb{R}^{3}$ where $s=-\ln |\mu|, t=\arg \frac{\bar{\mu}-1}{\bar{\mu}(1-\mu)}$. Moreover, $f_{j}$ and $f_{j}^{*}$ are the coordinates of $f=i f_{1}+j f_{2}+k f_{3}$ and $f^{*}=i f_{1}^{*}+j f_{2}^{*}+k f_{3}^{*}$ respectively. Since any simple factor dressing $\hat{f}$ of $f$ with parameters $(\mu, m, n)$ is given by a simple factor dressing with parameter $\mu$ and an operation of $\mathcal{R}_{n, m} \in \mathrm{SO}(4, \mathbb{R})$, we see from (34) that $\hat{f}$ is in 3 -space if $\mathcal{R}_{n, m}$ stabilises $\mathbb{C}=\operatorname{span}_{\mathbb{R}}\{1, i\}$. In particular:

Theorem 6.7 Let $f: M \rightarrow \mathbb{R}^{3}$ be minimal. The simple factor dressing $\hat{f}$ with parameters $(\mu, m, n)$ with $m=n \lambda, \lambda \in \mathbb{C}_{*}$, is a minimal surface $\hat{f}: M \rightarrow \mathbb{R}^{3}$ in 3-space.

As before, we also obtain the periods of the simple factor dressing:

Corollary 6.8 If $f: M \rightarrow \mathbb{R}^{3}$ is a periodic minimal surface with $\gamma^{*} f=f+\tau$ for $\gamma \in$ $\pi_{1}(M), \tau=\left(\tau_{1}, \tau_{2}, \tau_{3}\right)$, then the simple factor dressing $f^{\mu}$ of $f$ with parameter $\mu$ is periodic with $\gamma^{*} f^{\mu}=f^{\mu}+\tau^{\mu}$ where

$$
\tau^{\mu}=\left(\begin{array}{c}
\tau_{1} \\
\cos t\left(\tau_{2} \cosh s-\tau_{3}^{*} \sinh s\right)-\sin t\left(\tau_{3} \cosh s+\tau_{2}^{*} \sinh s\right) \\
\sin t\left(\tau_{2} \cosh s-\tau_{3}^{*} \sinh s\right)+\cos t\left(\tau_{3} \cosh s+\tau_{2}^{*} \sinh s\right)
\end{array}\right) .
$$

Here $\tau^{*}=\left(\tau_{1}^{*}, \tau_{2}^{*}, \tau_{3}^{*}\right)$ denote the periods of a conjugate surface $f^{*}$ of $f$, that is, $\gamma^{*} f^{*}=f^{*}+\tau^{*}$, and $s=-\ln |\mu|, t=\arg \frac{\bar{\mu}-1}{\bar{\mu}(1-\mu)}$.

In particular, $f^{\mu}$ is closed along $\gamma$ if and only if $\tau_{1}=0$ and $\left(\begin{array}{c}\tau_{2} \\ \tau_{3}\end{array}\right)=\left(\begin{array}{c}\tau_{3}^{*} \\ -\tau_{2}^{*}\end{array}\right) \tanh s$.

We can also investigate the behaviour of simple factor dressings in $\mathbb{R}^{3}$ at ends:

Theorem 6.9 Let $f: M \rightarrow \mathbb{R}^{3}$ be a minimal surface on a punctured disc $M=D \backslash\{p\}$ and $\hat{f}: \tilde{M} \rightarrow \mathbb{R}^{3}$ its simple factor dressing with parameter $(\mu, m, m), m \in S^{3}$.

Then the following hold:

(i) If $f$ has a planar end at $p$ then $\hat{f}: M \rightarrow \mathbb{R}^{3}$ is single-valued on $M$ and $\hat{f}$ has a planar end at $p$.

(ii) If $f$ has a catenoidal end at $p$ and $\hat{f}: M \rightarrow \mathbb{R}^{3}$ is single-valued on $M$ then $\hat{f}$ has a catenoidal end at $p$.

Proof Let $p$ be an complete, embedded, finite total curvature end of $f$. We can assume that the end of $f$ at $p$ is vertical: if the end is not vertical, let $n \in \mathbb{H}_{*}$ such that $\tilde{f}=\mathcal{R}_{n, n}^{-1} f$ has vertical normal at $p$. Since $\mathcal{R}_{m, m}=\mathcal{R}_{n, n} \circ \mathcal{R}_{n^{-1} m, n^{-1} m}$ and $\hat{f}=\mathcal{R}_{m, m}\left(\left(\mathcal{R}_{m, m}^{-1}(f)\right)^{\mu}\right)$ by Proposition 5.8, the simple factor dressing of $f$ is up to rotation given by the simple factor dressing of $\tilde{f}$ with parameters $\left(\mu, n^{-1} m, n^{-1} m\right)$. 
In a conformal coordinate $z$ on the punctured disk $D \backslash\{0\}$, we know from [38], see also Theorem 2.3, that the holomorphic null curve $\Phi$ of $f$ has $\operatorname{ord}_{z=0} d \Phi=-2$ and $\operatorname{res}_{z=0} d \Phi=$ $-(0,0,2 \pi \alpha)$ where $\alpha \in \mathbb{R}$ is the logarithmic growth.

By [38] the periods of the conjugate surface $f^{*}$ around the end are given by $\operatorname{res}_{z=0} d \Phi$. Therefore, if $f$ has a planar end then $f^{*}$ is single-valued on $M$, and if $f$ has a catenoidal end then the periods of $f^{*}$ are given by $-2 \pi \alpha k$. By Proposition 5.8 and Corollary 6.8, the simple factor dressing is single-valued for all parameters if $p$ is a planar end. Otherwise, it is single-valued for parameters $(\mu, m, m)$ such that $m^{-1} k m= \pm i$, that is, $m=(1 \mp j) \lambda$ with $\lambda \in \mathbb{C}$.

We know from Corollary 5.9 that the simple factor dressing preserves completeness, that is, the end of $\hat{f}$ at $p$ is complete. Since the simple factor dressing $\hat{f}$ is a Goursat transformation, the holomorphic null curve of $\hat{f}$ is given by $\hat{\Phi}=\mathcal{A} \Phi$ with $\mathcal{A} \in \mathrm{O}(3, \mathfrak{C})$ and thus, $\operatorname{ord}_{z=0} d \hat{\Phi}=-2$. At a planar end we have $\operatorname{res}_{z=0} d \hat{\Phi}=\operatorname{res}_{z=0} d \Phi=(0,0,0)$. Therefore, $\hat{f}$ has a planar end by Theorem 2.3 .

From Proposition 5.8 and (35) we know that $\mathcal{A}=\mathcal{R}_{m, m} \mathcal{L}^{\mu} \mathcal{R}_{m, m}^{-1}$ where

$$
\mathcal{L}^{\mu}=\left(\begin{array}{ccc}
1 & 0 & 0 \\
0 & \cosh w & \mathbf{i} \sinh w \\
0 & -\mathbf{i} \sinh w & \cosh w
\end{array}\right), \quad w=s+\mathbf{i} t .
$$

At a catenoidal end, a single-valued simple factor dressing has parameters $(\mu, m, m)$ with $m=(1 \mp j) \lambda, \lambda \in \mathbb{C}$. Thus, $\mathcal{R}_{m, m}^{-1} \operatorname{res}_{z=0} d \Phi$ is an eigenvector with eigenvalue 1 of the matrix $\mathcal{L}^{\mu}$. But then $\operatorname{res}_{z=0} d \hat{\Phi}=\operatorname{res}_{z=0} d \Phi$ is real, and the end of the simple factor dressing is catenoidal by Theorem 2.3 .

Again, we obtain from Theorem 6.3 the link to the López-Ros deformation:

Theorem 6.10 Let $f: M \rightarrow \mathbb{R}^{3}$ be a minimal surface in $\mathbb{R}^{3}$ with conjugate surface $f^{*}$. The López-Ros deformation with complex parameter $\sigma=e^{s+\mathbf{i} t} \in \mathfrak{C}_{*},|\sigma| \neq 1$, is the simple factor dressing of $f$ with parameters $(\mu, m, m)$ where $\mu=\frac{1-e^{-(s+i t)}}{1-e^{s-i t}} \in \mathbb{C}$ and $m=\frac{1-i-j-k}{2}$.

We obtain as a consequence of the last two theorems the following well-known result [48]:

Corollary 6.11 Let $f: M \rightarrow \mathbb{R}^{3}$ be a minimal surface on a punctured disk $M=D \backslash\{p\}$ and $f^{\sigma}: \tilde{M} \rightarrow \mathbb{R}^{3}$ a López-Ros deformation with parameter $\sigma$.

Then the following hold:

(i) If $f$ has a planar end at $p$ then $f^{\sigma}: M \rightarrow \mathbb{R}^{3}$ is single-valued on $M$ and $f^{\sigma}$ has a planar end at $p$.

(ii) If $f$ has a catenoidal end at $p$ and $f^{\sigma}: M \rightarrow \mathbb{R}^{3}$ is single-valued on $M$ then $f^{\sigma}$ has a catenoidal end at $p$.

Note that if $f$ is a minimal surface with vertical catenoidal end at $p$, then the proof of Theorem 6.9 shows that the López-Ros deformation is single-valued since $2 m=1-i-j-k=$ $(1-j)(1-i)$. In particular, the López-Ros deformation of $f$ has a catenoidal end at $p$, too.

\section{Examples}

We conclude this paper by demonstrating some of our results for well-known examples of minimal surfaces, including Richmond surfaces and the first Scherk surface. Further examples, such as the Riemann minimal examples and the Costa surface can be found in [45]. In 
particular, as we can control the periods and the end behaviour at punctures of simple factor dressings by choosing appropriate parameters, we obtain simple factor dressings which are minimal surfaces with one planar end and doubly-periodic surfaces respectively. Our first example is the catenoid for which all computations can be done completely explicitly. Berlin.

The images were implemented by using the software jReality and the jTEM library of TU

\subsection{The catenoid}

We consider the catenoid $f: \mathfrak{C} \rightarrow \mathbb{R}^{3}$ in the conformal parametrisation

$$
f(x, y)=i x+j \cosh x e^{-i y},
$$

where $z=x+i y$ is the standard conformal coordinate on $\mathbb{C}$ with $* d z=i d z$. The left and right normal of the catenoid are given by the Gauss map

$$
R(x, y)=N(x, y)=\frac{1}{\cosh x}\left(i \sinh x-j e^{-i y}\right) .
$$

A conjugate surface is the helicoid

$$
f^{*}(x, y)=i y+j i \sinh x e^{-i y}
$$

and, identifying $z=x+\mathbf{i} y$, we obtain the holomorphic null curve

$$
\Phi(z)=f(z)+\mathbf{i} f^{*}(z)=\left(\begin{array}{c}
z \\
\cosh z \\
-\mathbf{i} \sinh z
\end{array}\right): \mathbb{C} \rightarrow \mathfrak{C}^{3},
$$

and the Weierstrass data $g(z)=\frac{e^{z}-\mathbf{i}}{e^{z}+\mathbf{i}}$ and $\omega=-\frac{\mathbf{i}}{2} e^{-z}\left(e^{z}+\mathbf{i}\right)^{2} d z$. The right associated family $f_{p, q}=f p+f^{*} q$ is given (25) by

$$
f_{p, q}(x, y)=i(p x+q y)+j e^{-i y}(p \cosh x+i q \sinh x), \quad p, q \in \mathbb{H},
$$

and the left associated family $f^{p, q}=p f+q f^{*}$ by

$$
f^{p, q}(x, y)=(p x+q y) i+(p \cosh x-q i \sinh x) j e^{-i y}, \quad p, q \in \mathbb{H},
$$

see Figs. 1, 2.

The López-Ros deformation of the catenoid, see (Fig. 3), with parameter $\sigma=e^{s+\mathbf{i} t} \in \mathfrak{C}_{*}$ is given, see Theorem 2.4, by

$$
f_{\sigma}(x, y)=\left(\begin{array}{c}
\cos t(x \cosh s-\sinh x \sin y \sinh s)-\sin t(\cosh x \cos y \cosh s+y \sinh s) \\
\sin t(x \cosh s-\sinh x \sin y \sinh s)+\cos t(\cosh x \cos y \cosh s+y \sinh s) \\
\cosh x \sin y
\end{array}\right) .
$$

The periods of the simple factor dressing with parameter $\mu$ are given by Corollary 6.8 :

Lemma 7.1 The simple factor dressing $\hat{f}$ of the catenoid with parameters $(\mu, m, m)$ has translational periods

$$
\hat{f}(x, y+2 \pi)=\hat{f}(x, y)+\pi\left(i \hat{b}-\frac{\hat{b}}{\hat{a}-1} i(\hat{a}-1)\right),
$$

where $\hat{a}=m \frac{\mu+\mu^{-1}}{2} m^{-1}, \hat{b}=m i \frac{\mu^{-1}-\mu}{2} m^{-1}$. 

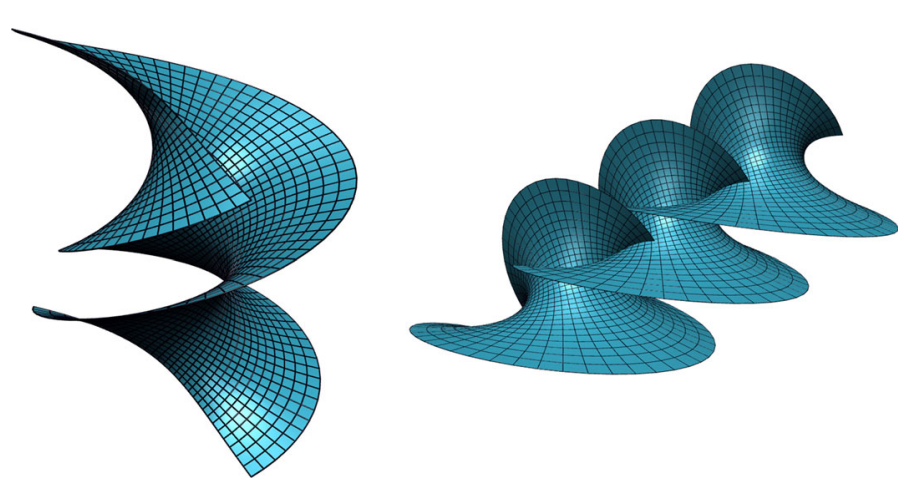

Fig. 1 Elements $f \frac{2}{\sqrt{6}}, \frac{1}{\sqrt{6}}+\frac{i}{\sqrt{6}}$ and $f \frac{1}{\sqrt{2}}, \frac{1}{2 \sqrt{2}}(1+i-j-k)$ of the right associated family of the catenoid, orthogonally projected into $\mathbb{R}^{3}$
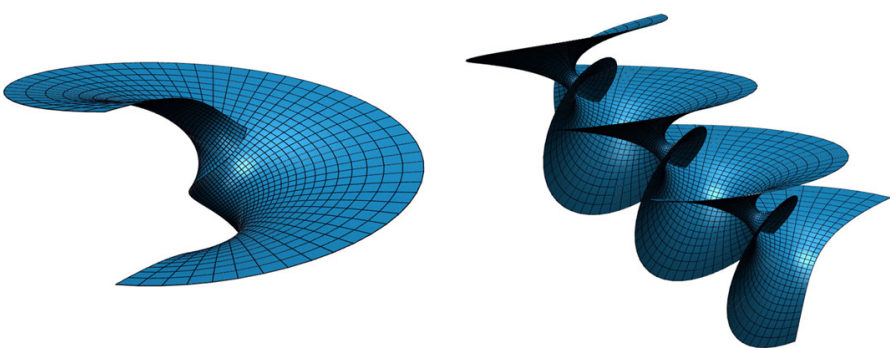

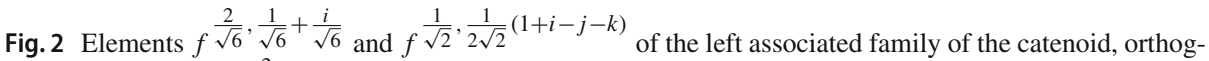
onally projected into $\mathbb{R}^{3}$
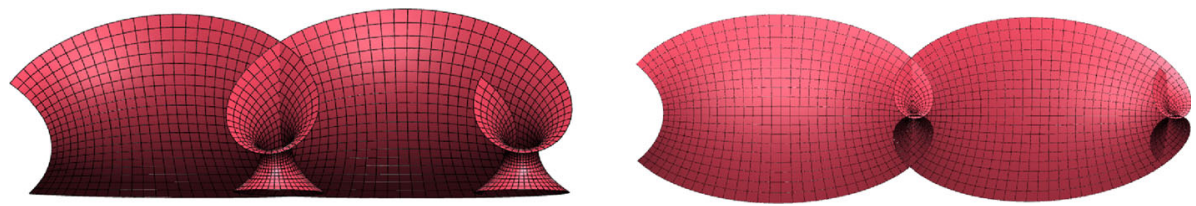

Fig. 3 The López-Ros deformation of the catenoid with parameter $\sigma=2$ and $\sigma=4$

In particular, $\hat{f}(x, y+2 \pi)=\hat{f}(x, y)$ if and only if $m \in \mathbb{C}_{*}$ or $m \in \mathbb{C}_{*} j$ or $\mu \in S^{1}$. In this case, $\hat{f}$ is a (reparametrised) catenoid.

Proof Since $\hat{f}=\mathcal{R}_{m, m}\left(\left(\mathcal{R}_{m, m}^{-1}(f)\right)^{\mu}\right)$ is the simple factor dressing with parameters $(\mu, m, m)$ it is enough to investigate the periods of the simple factor dressing $\tilde{f}^{\mu}$ with parameter $\mu$ of the minimal surface $\tilde{f}=\mathcal{R}_{m, m}^{-1} f$. Since $\tilde{f}(x, y+2 \pi)=\tilde{f}(x, y)$ and $\tilde{f}^{*}(x, y+2 \pi)=\tilde{f}^{*}(x, y)+2 \pi m^{-1}$ im we see by Corollary 6.8 that the simple factor dressing $\tilde{f}^{\mu}$ has vanishing periods if and only if $\mu \in S^{1}$ or $m^{-1} i m= \pm i$, that is, $m \in \mathbb{C}_{*}$ or $m \in \mathbb{C}_{*} j$. In the former case, the simple factor dressing of $f$ is trivial. In the latter case we see with Theorem 5.5 that $\hat{f}$ is the simple factor dressing of $f$ with parameter $\mu$ or $\bar{\mu}^{-1}$. But the simple factor dressing of $f$ with parameter $\mu$ is by (37) given by

$$
f^{\mu}(x, y)=i x+j \cosh (x+s) e^{-i(y+t)}
$$



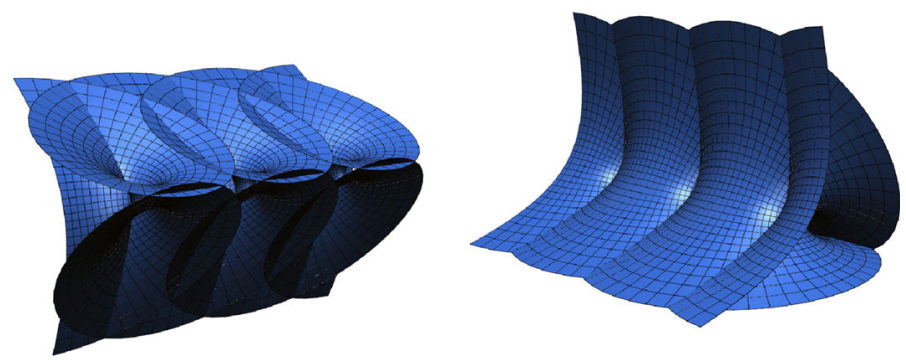

Fig. 4 Simple factor dressing of the catenoid with parameters $\left(-\frac{i}{2}, \frac{1}{2}(1+i-j-k)\right)$
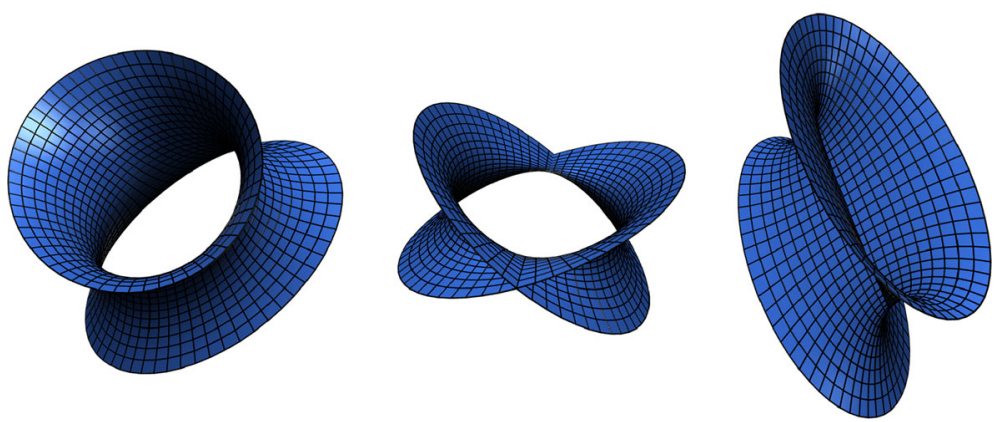

Fig. 5 Simple factor dressing of the catenoid with parameters $\left(-\frac{i}{2}, \frac{1+k}{2}, \frac{i-j}{2}\right)$, various orthogonal projections to $\mathbb{R}^{3}$

where $s=-\ln |\mu|, t=\arg \frac{\bar{\mu}-1}{\bar{\mu}(1-\mu)}$. Thus, for $m \in \mathbb{C}_{*}$ we see that $\hat{f}=f^{\mu}$ is a reparametrisation of the catenoid. Using again Theorem 5.5 we obtain also the case $m \in \mathbb{C}_{*} j$.

In the case of general parameters $(\mu, m, m), m \in \mathbb{H}_{*}$, we obtain with (32) that

$$
\hat{f}(x, y+2 \pi)=\hat{f}(x, y)+\pi\left(i \hat{b}-\frac{\hat{b}}{\hat{a}-1} i(\hat{a}-1)\right) .
$$

Thus, in general the simple factor dressing of a catenoid will have translational periods. Although the resulting surfaces resemble Catalan's surface see (Fig. 4) the simple factor dressing of a catenoid has by Corollary 5.9 no branch points.

If we allow the simple factor dressing to be a minimal surface in $\mathbb{R}^{4}$ we obtain with Corollary 6.5 further closed minimal surfaces: for example, when choosing $m=\frac{1+k}{2}, n=$ $\frac{i-j}{2}$ then the simple factor dressing with parameters $(\mu, m, n)$ gives a minimal immersion into $\mathbb{R}^{4}$ by

$$
\hat{f}(x, y)=i x+k \sin y \cosh x+(\sin y \sinh x \sinh s+j \cos y \cosh x \cosh s) e^{-j t},
$$

where $s=-\ln |\mu|, t=\arg \frac{\bar{\mu}-1}{\bar{\mu}(1-\mu)}$. In particular, we immediately see that $\hat{f}(x, y+2 \pi)=$ $\hat{f}(x, y)$ (Fig. 5).

\subsection{Richmond surfaces}

We will now consider examples [41], of Richmond surfaces which are minimal surfaces with one planar end given by the Weierstrass data $g(z)=z^{l+1}, d h=z^{l-1} d z, l \in \mathbb{N}$, that is, 

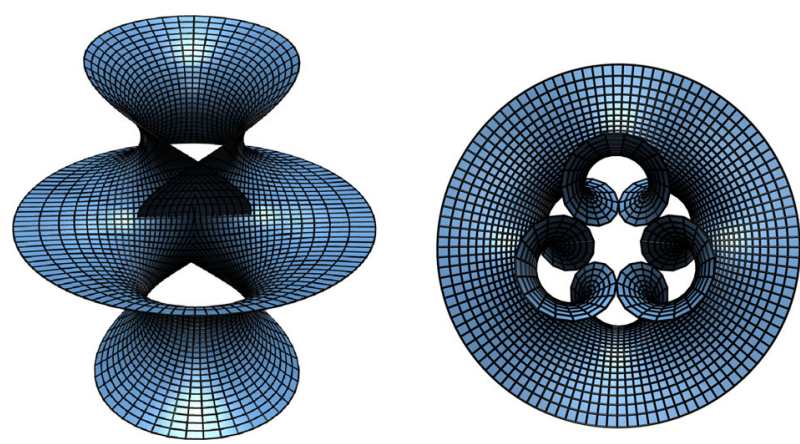

Fig. 6 Minimal surfaces with one planar end, $l=1$ and $l=3$

$f(x, y)=\operatorname{Re} \Phi(z), z=x+i y \in \mathbb{C}_{*}$, where the holomorphic null curve $\Phi$ is given, see (6), as

$$
\Phi(z)=\left(\frac{1}{2}\left(-\frac{1}{z}-\frac{z^{2 l+1}}{2 l+1}\right) \frac{\mathbf{i}}{2}\left(-\frac{1}{z}+\frac{z^{2 l+1}}{2 l+1}\right), \frac{z^{l}}{l}\right): \mathbb{C}_{*} \rightarrow \mathfrak{C}^{3} .
$$

Here we identify as before $z=x+\mathbf{i} y$. Indeed, by Theorem 2.3 the immersion $f$ has a planar end at the puncture $z=0$ since ord $\operatorname{or}_{z=0} d \Phi=-2$ and the residue of $d \Phi$ at $z=0$ vanishes (Fig. 6).

Then the conjugate surface

$$
f^{*}(x, y)=\operatorname{Im} \Phi(z)
$$

is single-valued on $\mathbb{C}_{*}$, and so are the left and right associated family (Fig. 7).

We discuss the simple factor dressings of $f$ with parameter $\mu$ in more detail for the case $l=1$, that is,

$$
\begin{aligned}
f(x, y)= & -\frac{i x}{2}\left(\frac{1}{x^{2}+y^{2}}+\frac{x^{2}-3 y^{2}}{3}\right) \\
& -\frac{j y}{2}\left(\frac{1}{x^{2}+y^{2}}+\frac{3 x^{2}-y^{2}}{3}\right)+k x, \quad(x, y) \neq(0,0),
\end{aligned}
$$

with Gauss map, using (7),

$$
\begin{aligned}
N(x, y)= & \frac{1}{1+\left(x^{2}+y^{2}\right)^{2}}\left(2 i\left(x^{2}-y^{2}\right)+4 j x y\right. \\
& +k\left(\left(x^{2}+y^{2}\right)^{2}-1\right), \quad(x, y) \neq(0,0),
\end{aligned}
$$

and conjugate minimal surface

$$
\begin{aligned}
f^{*}(x, y)= & \frac{i y}{2}\left(\frac{1}{x^{2}+y^{2}}+\frac{y^{2}-3 x^{2}}{3}\right) \\
& -\frac{j x}{2}\left(\frac{1}{x^{2}+y^{2}}+\frac{3 y^{2}-x^{2}}{3}\right)+k y, \quad(x, y) \neq(0,0) .
\end{aligned}
$$



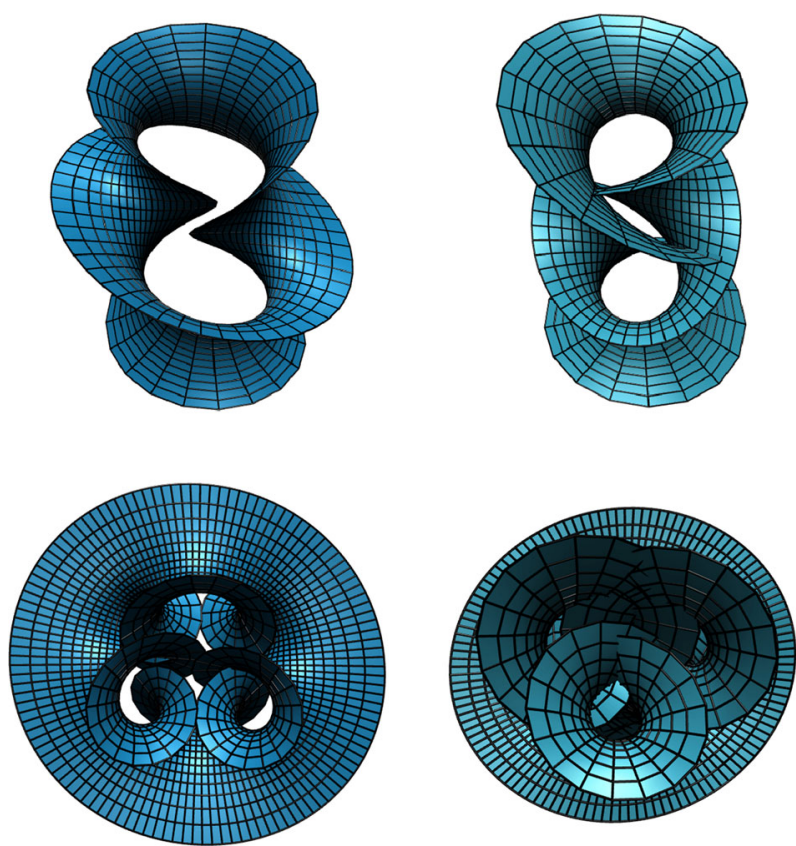

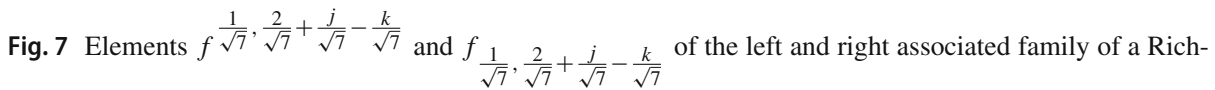
mond surface, $l=1$ and $l=3$, orthogonally projected into $\mathbb{R}^{3}$

The simple factor dressing $f^{\mu}$ with parameter $\mu$ is given (37) by

$$
\begin{aligned}
f^{\mu}(x, y)=- & \frac{i x}{2}\left(\frac{1}{x^{2}+y^{2}}+\frac{x^{2}-3 y^{2}}{3}\right) \\
+ & \left\{-j y\left(\frac{1}{2}\left(\frac{1}{x^{2}+y^{2}}+\frac{3 x^{2}-y^{2}}{3}\right) \cosh s+\sinh s\right)\right. \\
& \left.+k x\left(\cosh s-\frac{1}{2}\left(\frac{1}{x^{2}+y^{2}}+\frac{3 y^{2}-x^{2}}{3}\right) \sinh s\right)\right\} e^{-i t, \quad(x, y) \neq(0,0),}
\end{aligned}
$$

where $s=-\ln |\mu|, t=\arg \frac{\bar{\mu}-1}{\bar{\mu}(1-\mu)}$. From Theorem 6.9 we know that $f^{\mu}$ is a minimal surface with a planar end at the puncture $(x, y)=(0,0)$.

We recall that $\mu=\frac{1-e^{-(s+i t)}}{1-e^{s-i t}}$ so that $\rho=i \frac{1+\mu}{1-\mu}=i \frac{1+e^{2 s}-2 e^{s+i t}}{e^{2 s}-1}$. Since $N^{\mu}=(N+$ $\rho) N(N+\rho)^{-1}$ and $\lim _{(x, y) \rightarrow(0,0)} N(x, y)=-k$ we therefore see that

$$
\lim _{(x, y) \rightarrow(0,0)} N^{\mu}(x, y)=(k-\rho) k(\rho-k)^{-1}=\frac{1}{\cosh (s)}\left(i \sinh (s)-k e^{-i t}\right) .
$$

In particular, the end near the puncture $(x, y)=(0,0)$ is asymptotic to the plane spanned by $\left(i+k \sinh s e^{-i t}\right)$ and $j e^{-i t}$ (Fig. 8).

By Theorem 2.4 the López-Ros deformation of $f$ with parameter $\sigma=e^{s+i t} \in \mathfrak{C}_{*}$ is 

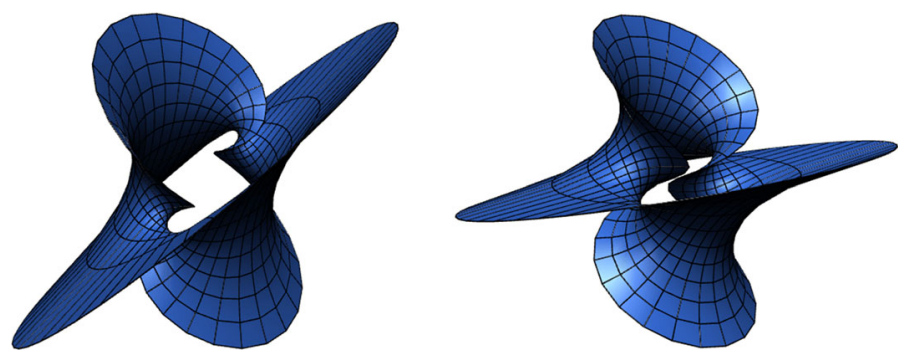

Fig. 8 Simple factor dressing of a Richmond surface, $l=1$, with parameters $\mu=-\frac{i}{2}$ and $\mu=-\frac{1}{2}+\frac{i}{2}$
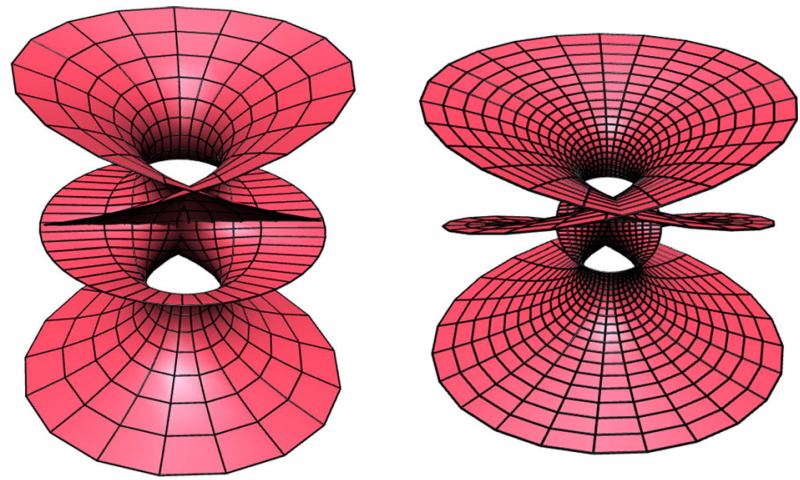

Fig. 9 López-Ros deformation of a Richmond surface, $l=1$, with parameters $\sigma=2$ and $\sigma=7$
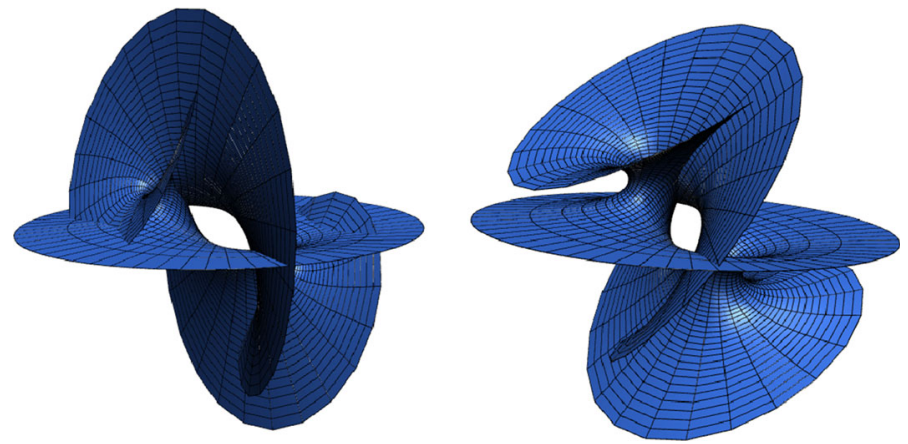

Fig. 10 Simple factor dressing of a Richmond surface, $l=1$, with parameters $(\mu, m, m)$ with $\mu=-\frac{i}{2}$ and $\mu=-\frac{1}{2}+\frac{i}{2}, m=\frac{1}{2}-k$

$$
f_{\sigma}=\left(\begin{array}{c}
x \cos t\left(-\frac{1}{x^{2}+y^{2}} e^{-s}+\frac{3 y^{2}-x^{2}}{3} e^{s}\right)-y \sin t\left(-\frac{1}{x^{2}+y^{2}} e^{-s}+\frac{y^{2}-3 x^{2}}{3} e^{s}\right) \\
x \sin t\left(-\frac{1}{x^{2}+y^{2}} e^{-s}+\frac{3 y^{2}-x^{2}}{3} e^{s}\right)+y \cos t\left(-\frac{1}{x^{2}+y^{2}} e^{-s}+\frac{y^{2}-3 x^{2}}{3} e^{s}\right) \\
x
\end{array}\right)
$$

$f_{\sigma}$ has a vertical planar end at the puncture $z=x+i y=0$. From the holomorphic null curves of $f$ and $f_{\sigma}, \sigma \neq 1$, we see that $f_{\sigma}$ is not a reparametrisation of $f$ (Fig. 9). 

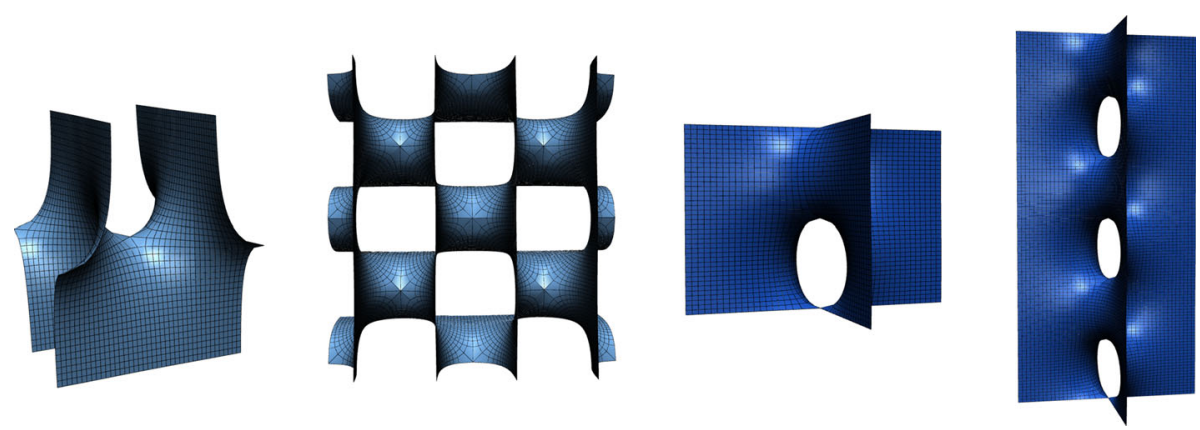

Fig. 11 Scherk's first surface and its conjugate, Scherk's fifth surface (parametrisation by height)
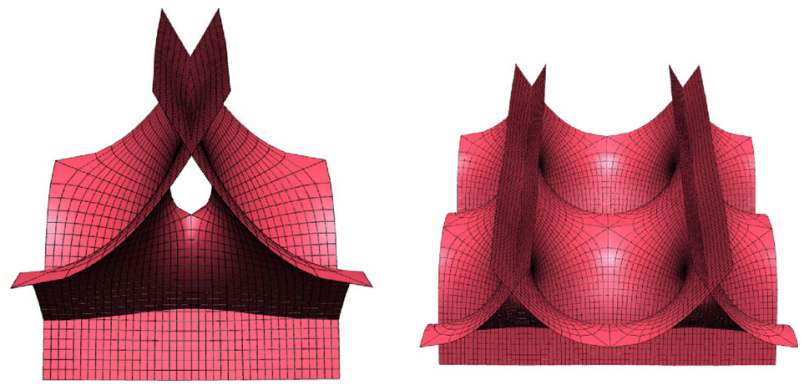

Fig. 12 López-Ros deformation of Scherk's first surface with parameter $\sigma=0.6$

Finally, we include some pictures of the simple factor dressing for more general parameters. Note that the surfaces are single-valued for all parameters $(\mu, m, m)$, and have a planar end at $z=0$ (Fig. 10).

\subsection{Scherk surfaces}

We will now consider the first Scherk surface given by the Weierstrass data $g(z)=z, \omega(z)=$ $-\frac{4}{z^{4}-1} d z$, that is, $f=\operatorname{Re} \Phi$ is the real part of the (multi-valued) holomorphic null curve

$$
\Phi(z)=\left(\mathbf{i} \log \frac{z+\mathbf{i}}{z-\mathbf{i}}, \mathbf{i} \log \frac{z+1}{z-1}, \log \frac{z^{2}+1}{z^{2}-1}\right), z \in \mathbb{C} \backslash\{ \pm 1, \pm i\},
$$

identifying again $z=x+\mathbf{i} y$ (Fig. 11).

Denoting by $\gamma_{p}$ the positively oriented circle around $p \in\{ \pm 1, \pm i\}$ the periods $\gamma_{p}^{*} \Phi=$ $\Phi+\tau_{p}+\mathbf{i} \tau_{p}^{*}$ of $\Phi$ are given by

$$
\tau_{ \pm 1}+\mathbf{i} \tau_{ \pm 1}^{*}=2 \pi(0, \pm 1,-\mathbf{i}), \quad \tau_{ \pm i}+\mathbf{i} \tau_{ \pm i}^{*}=2 \pi( \pm 1,0, \mathbf{i}) .
$$

In particular, the doubly-periodic first Scherk surface has periods $\gamma_{p}^{*} f=f+\tau_{p}$ and its conjugate, the simply-periodic fifth Scherk surface, has periods $\gamma_{p}^{*} f^{*}=f^{*}+\tau_{p}^{*}$.

Since $\tau_{p}^{*} \in \operatorname{span}_{\mathbb{R}}\{k\}$ we see with Theorem 2.4 that the López-Ros deformation of $f$ with parameter $\sigma$ is doubly-periodic (Figs. 12,13) with 

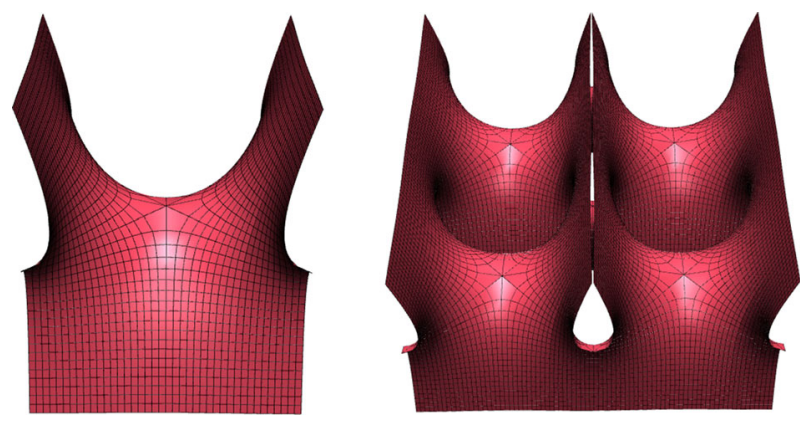

Fig. 13 López-Ros deformation of Scherk's first surface with parameter $\sigma=1.4$
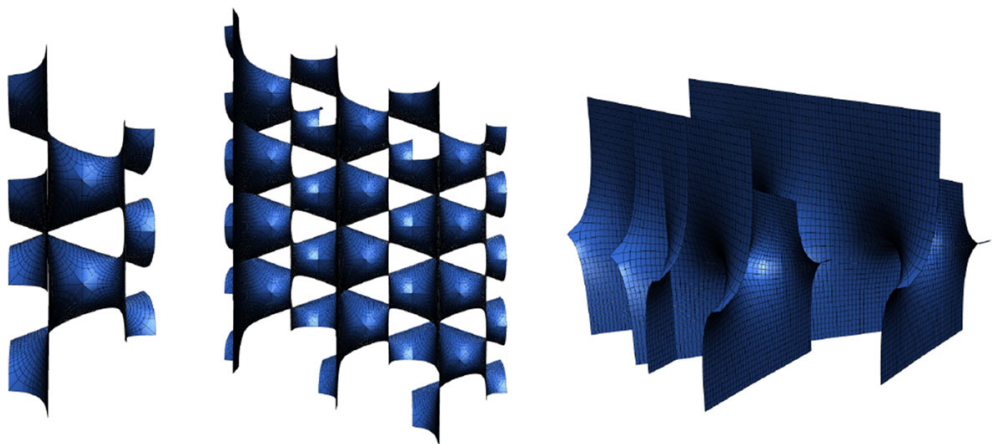

Fig. 14 Simple factor dressing with $\mu=-\frac{1}{\sqrt{2}}$ of Scherk's first surface: fundamental domain for the lattice $\Gamma=<2 \pi \sqrt{2} j, 2 \pi\left(i-\frac{\sqrt{2} j}{4}\right)>$, larger piece of the surface, and side view

$$
\gamma_{p}^{*} f_{\sigma}=f_{\sigma}+\left(\begin{array}{ccc}
\cos t & -\sin t & 0 \\
\sin t & \cos t & 0 \\
0 & 0 & 1
\end{array}\right) \tau_{p} \cosh s .
$$

Corollary 6.8 shows that the periods of the simple factor dressing with parameter $\mu$ are given by

$$
\tau_{ \pm 1}^{\mu}= \pm 2 \pi e^{ \pm s}\left(\begin{array}{c}
0 \\
\cos t \\
\sin t
\end{array}\right), \quad \tau_{ \pm i}^{\mu}=2 \pi\left(\begin{array}{c} 
\pm 1 \\
-\cos t \sinh s \\
-\sin t \sinh s
\end{array}\right),
$$

where $s=-\ln |\mu|, t=\arg \frac{\bar{\mu}-1}{\bar{\mu}(1-\mu)}$; in particular, the periods cannot be simultaneously closed. Moreover, since $\tau_{1}^{\mu}+\tau_{-1}^{\mu}+\tau_{i}^{\mu}+\tau_{-i}^{\mu}=0$ and $\tau_{1}^{\mu}=-\tau_{-1}^{\mu} e^{2 s}$ we see that $f^{\mu}$ is doubly-periodic with respect to the integer lattice generated by $b \tau_{1}^{\mu}$ and $\tau_{i}^{\mu}$ if $s=\ln \sqrt{q}$ with $q=\frac{a}{b}, a, b \in \mathbb{N}$.

In particular, for $q \in \mathbb{N}$ and $\mu=-\frac{1}{\sqrt{q}}$ the simple factor dressing with parameter $\mu$ is invariant under the integer lattice $\Gamma=<\tau_{1}^{\mu}, \tau_{i}^{\mu}>$ since $q=\frac{a}{b}$ with $a=q, b=1$, and thus $b \tau_{1}^{\mu}=\tau_{1}^{\mu}$ (Fig. 14).

However, as already indicated by the pictures above, in this case a simple factor dressing is invariant under a smaller lattice: since $\tau_{1}^{\mu}=-q \tau_{-1}^{\mu}$ with $q \in \mathbb{N}$, we see that the simple factor dressing is invariant under the integer lattice $\hat{\Gamma}=<\tau_{-1}^{\mu}, \tau_{i}^{\mu}>$ (Fig. 15). 

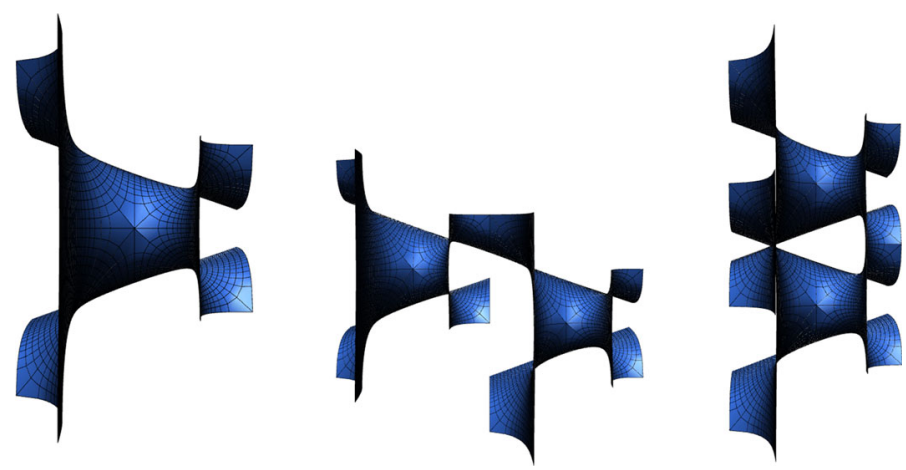

Fig. 15 Simple factor dressing with $\mu=-\frac{1}{\sqrt{2}}$ of Scherk's first surface: fundamental domain for the lattice $\hat{\Gamma}=<\tau_{i}^{\mu}, \tau_{-1}^{\mu}>$, translations by $\tau_{i}^{\mu}=2 \pi\left(i-\frac{\sqrt{2} j}{4}\right)$ and $\tau_{-1}^{\mu}=-\pi \sqrt{2} j$
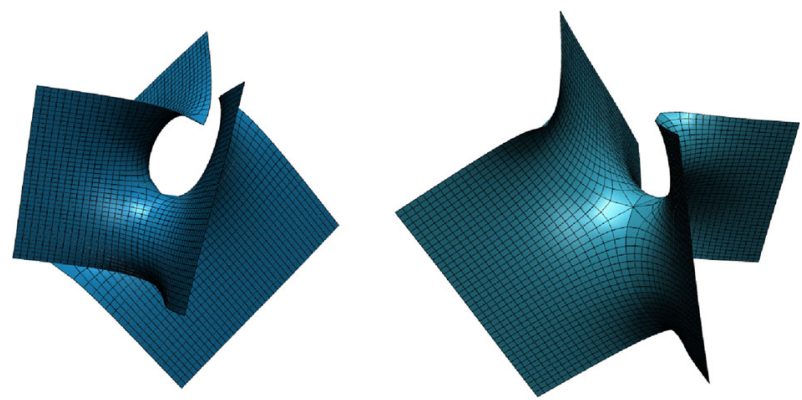

Fig. 16 Elements $f^{\frac{1}{\sqrt{7}}, \frac{2}{\sqrt{7}}+\frac{j}{\sqrt{7}}-\frac{k}{\sqrt{7}}}$ and $f_{\frac{1}{\sqrt{7}}, \frac{2}{\sqrt{7}}+\frac{j}{\sqrt{7}}-\frac{k}{\sqrt{7}}}$ of the left and right associated family of Scherk's first surface, orthogonally projected into $\mathbb{R}^{3}$

We conclude the example of Scherk's first surface by providing the pictures for the leftand right-associated family which give minimal surfaces in $\mathbb{R}^{4}$ (Fig. 16).

Open Access This article is distributed under the terms of the Creative Commons Attribution 4.0 International License (http://creativecommons.org/licenses/by/4.0/), which permits unrestricted use, distribution, and reproduction in any medium, provided you give appropriate credit to the original author(s) and the source, provide a link to the Creative Commons license, and indicate if changes were made.

\section{References}

1. Bernstein, J., Breiner, C.: Symmetry of embedded genus 1 helicoids. Duke Math. J. 159, 83-97 (2011)

2. Burstall, F.E.: Isothermic surfaces: conformal geometry, Clifford algebras and integrable systems. In: Integrable Systems, Geometry, and Topology, AMS/IP Stud. Adv. Math., vol. 36, pp. 1-82. Amer. Math. Soc., Providence (2006)

3. Burstall, F.E., Pedit, F.: Dressing orbits of harmonic maps. Duke Math. J. 80(2), 353-382 (1995)

4. Burstall, F., Quintino, A.: Dressing transformations of constrained Willmore surfaces. Commun. Anal. Geom. 22, 469-518 (2014)

5. Burstall, F.E., Ferus, D., Pedit, F., Pinkall, U.: Harmonic tori in symmetric spaces and commuting Hamiltonian systems on loop algebras. Ann. Math. 138, 173-212 (1993) 
6. Burstall, F.E., Hertrich-Jeromin, U., Pedit, F., Pinkall, U.: Curved flats and isothermic surfaces. Math. Z. 225, 199-209 (1997)

7. Burstall, F., Ferus, D., Leschke, K., Pedit, F., Pinkall, U.: Conformal Geometry of Surfaces in $S^{4}$ and Quaternions. Lecture Notes in Mathematics. Springer, Berlin (2002)

8. Burstall, F., Pedit, F., Pinkall, U.: Schwarzian derivatives and flows of surfaces. In: Ohnita, Y., Guest, M., Miyaoka, R. (eds.) Differential Geometry and Integrable Systems, Contemporary Mathematics, vol. 308, pp. 39-61. AMS, Providence (2002)

9. Burstall, F., Dorfmeister, J., Leschke, K., Quintino, A.: Darboux transforms and simple factor dressing of constant mean curvature surfaces. Manuscr. Math. 140(1), 213-236 (2013)

10. Bobenko, A.: Constant mean curvature surfaces and integrable equations. Russ. Math. Surv. 40, 1-45 (1991)

11. Bohle, C.: Constrained Willmore tori in the 4-sphere. J. Differ. Geom. 86(1), 71-132 (2010)

12. Bohle, C., Taimanov, I.A.: Euclidean minimal tori with planar ends and elliptic solitons. Int. Math. Res. Not., pp. 1-26 (2014)

13. Bohle, C., Leschke, K., Pedit, F., Pinkall, U.: Conformal maps from a 2-torus to the 4-sphere. J. Reine Angew. Math. 671, 1-30 (2012)

14. Bonnet, O.: Note sur la théorie générale des surfaces. Comptes rendus des Séances de l'Académie des Sciences 37, 529-532 (1853)

15. Carberry, E., Leschke, K., Pedit, F.: Darboux transforms and spectral curves of constant mean curvature surfaces revisited. Ann. Glob. Anal. Geom. 43(4), 299-329 (2013)

16. Cayley, A.: On certain results relating to quaternions. Math. Papers 1, 123-126 (1889)

17. Chern, S., Osserman, R.: Complete minimal surfaces in Euclidean n-space. J. Anal. Math. 19(1), 15-34 (1967)

18. Cieśliński, J., Goldstein, P., Sym, A.: Isothermic surfaces in $E^{3}$ as soliton surfaces. Phys. Lett. A 205(1), 37-43 (1995)

19. Dierkes, U., Hildebrandt, S., Sauvigny, F.: Minimal Surfaces. Springer, Berlin (2010)

20. Dorfmeister, J., Kilian, M.: Dressing preserving the fundamental group. Differ. Geom. Appl. 23, 176-204 (2005)

21. Dorfmeister, J., Pedit, F., Wu, H.: Weierstrass type representation of harmonic maps into symmetric spaces. Commun. Anal. Geom. 6(4), 633-667 (1998)

22. Dorfmeister, J., Pedit, F., Toda, M.: Minimal surfaces via loop groups. Balkan J. Geom. Appl. 2, 25-40 (2007)

23. Eells, J., Lemaire, L.: A report on harmonic maps. Bull. Lond. Math. Soc. 10, 1-68 (1978)

24. Eells, J., Lemaire, L.: Another report on harmonic maps. Bull. Lond. Math. Soc. 20, $385-524$ (1988)

25. Eisenhart, L.: Minimal surfaces in Euclidean four-space. Am. J. Math. 34(3), 215-236 (1912)

26. Ejiri, N.: Willmore surfaces with a duality in $S^{n}$ (1). Proc. Lond. Math. Soc. III Ser. 57(2), 383-416 (1988)

27. Enneper, A.: Analytisch-geometrische Untersuchungen. Z. Math. Phys. 9, 96-125 (1864)

28. Ferrer, L., Martin, F., Umehara, M., Yamada, K.: A construction of a complete bounded null curve in $C^{3}$. Kodai Math. J. 37, 59-96 (2014)

29. Ferus, D., Leschke, K., Pedit, F., Pinkall, U.: Quaternionic holomorphic geometry: Plücker formula, Dirac eigenvalue estimates and energy estimates of harmonic 2-tori. Invent. Math. 146, 507-593 (2001)

30. Goursat, E.: Sur un mode de transformation des surfaces minima. Acta Math. 11, 135-186 (1887)

31. Guest, M.A.: Harmonic Maps, Loop Groups, and Integrable Systems. Cambridge University Press, Cambridge (1997)

32. Hauswirth, L., Kilian, M., Schmidt, M.: Properly embedded minimal annuli in $S^{2} \times \mathbb{R}$. arXiv:1210.5953 (2013)

33. Hauswirth, L., Kilian, M., Schmidt, M.: The geometry of embedded constant mean curvature tori in the 3-sphere via integrable systems. Proc. Lond. Math. Soc. 112(3), 588-622 (2016)

34. Hélein, F.: Willmore immersions and loop groups. J. Differ. Geom. 50(2), 331-385 (1998)

35. Hélein, F., Romon, P.: Hamiltonian stationary Lagrangian surfaces in $\mathbb{C}^{2}$. Commun. Anal. Geom. 10(1), 79-126 (2002)

36. Hélein, F., Wood, J.C: Harmonic maps. In: Handbook of Global Analysis, pp. 41749. Elsevier Sci. B. V. (2008)

37. Hitchin, N.: Harmonic maps from a 2-torus to the 3-sphere. J. Differ. Geom. 31(3), 627-710 (1990)

38. Hoffman, D., Karcher, H.: Complete Embedded Minimal Surfaces of Finite Total Curvature. Springer, Berlin (1997)

39. Hoffman, D., Osserman, R.: The geometry of the generalized Gauss map. Mem. Am. Math. Soc. 28(236), iii+105pp (1980)

40. Jorge, L.P., Meeks III, W.H.: The topology of complete minimal surfaces of finite total Gaussian curvature. Topology 22(2), 203-221 (1983) 
41. Karcher, H.: Construction of minimal surfaces. Surveys in Geometry, Lecture Notes No. 12, SFB256, p. 196. University of Tokyo, Bonn (1989)

42. Lagrange, J.: Essai d'une nouvelle méthode pour déterminer les maxima et les minima des formules intégrales indéfinies. Misc. Taur. 2, 173-195 (1760)

43. Leschk, K.: Harmonic map methods for Willmore surfaces. Contemporary Mathematics, vol. 542, pp. 204-212. AMS, Providence (2011)

44. Leschke, K.: Generalised Darboux transforms of isothermic surfaces (2018) (in preparation)

45. Leschke, K., Moriya, K.: Applications of quaternionic holomorphic geometry to minimal surfaces. Complex Manifolds 3(1), 282-300 (2016)

46. Leschke, K., Romon, P.: Spectral curve of Hamiltonian stationary tori. Calc. Var. Partial Differ. Equ. 38(1), 45-74 (2010)

47. López, F., Martin, F.: Complete minimal surfaces in $R^{3}$. Publ. Mat. 43(2), 341-449 (1999)

48. López, F.J., Ros, A.: On embedded complete minimal surfaces of genus zero. J. Differ. Geom. 33(1), 293-300 (1991)

49. Meeks III, W.H., Pérez, J.: Properly embedded minimal planar domains with infinite topology are Riemann minimal examples. In: Current Developments in Mathematics, vol. 2008, pp. 281-346. International Press (2009)

50. Meeks III, W.H., Pérez, J.: A survey on classical minimal surface theory. University Lecture Series (AMS), vol. 60 (2012)

51. Meeks III, W.H., Pérez, J., Ros, A.: The geometry of minimal surfaces of finite genus I; curvature estimates and quasiperiodicity. J. Differ. Geom. 66, 1-45 (2004)

52. Meeks III, W.H., Pérez, J., Ros, A.: Properly embedded minimal planar domains. Ann. Math. 181(3), 473-546 (2014)

53. Mikhailov, A.V., Zakharov, V.E.: Relativistically invariant two-dimensional models in field theory integrable by the inverse scattering problem method. JETP Lett. 47, 1017-1027 (1978)

54. Moriya, K.: On a variety of algebraic minimal surfaces in Euclidean 4-space. Tokyo J. Math. 21(1), 121-134 (1998)

55. Nitsche, J.C.C.: Lectures on Minimal Surfaces, vol. 1. Introduction, Fundamentals, Geometry and Basic Boundary Value Problems. Cambridge University Press, Cambridge (1989)

56. Ohnita, Y.: Harmonic maps of surfaces and integrable system approach (a survey). The progress and view of Harmonic Map Theory (Kyoto, 2010). ūrikaisekikenkyūsho Kōkyūroku 1720, 1-27 (2010)

57. Osserman, R.: Global properties of minimal surfaces in $E^{3}$ and $E^{n}$. Ann. Math. 2(80), 340-364 (1964)

58. Pérez, J., Ros, A.: Properly embedded minimal surfaces with finite total curvature. In: The Global Theory of Minimal Surfaces in Flat Spaces, Lecture Notes in Mathematics, vol. 1775, pp. 15 - 66. Springer, Berlin (2002)

59. Pinkall, U., Sterling, I.: On the classification of constant mean curvature tori. Ann. Math. 130, 407-451 (1989)

60. Pohlmeyer, K.: Integrable Hamiltonian systems and interactions through quadratic constraints. Commun. Math. Phys. 46(3), 207-221 (1976)

61. Quintino, A.: Constrained Willmore surfaces: symmetries of a Möbius invariant integrable system. arXiv:0912.5402 (2009)

62. Rigoli, M.: The conformal Gauss map of submanifolds of the Moebius space. Ann. Glob. Anal. Geom. 5(2), 97-116 (1987)

63. Ruh, E., Vilms, J.: The tension field of the Gauss map. Trans. Am. Math. Soc. 149, 569-573 (1970)

64. Schmidt, M.: A proof of the Willmore conjecture. arXiv:math/0203224 (2002)

65. Schoen, R.: Uniqueness, symmetry, and embeddedness of minimal surfaces. J. Differ. Geom. 18, 791-809 (1983)

66. Schwarz, H.A.: Gesammelte Mathematische Abhandlungen, vol. 1. Springer, Berlin (1890)

67. Shabat, A., Zakharov, V.: Integration of nonlinear equations of mathematical physics by the method of inverse scattering. II. Funct. Anal. Appl. 13(3), 166-174 (1979)

68. Taimanov, I.A.: The Weierstrass representation of closed surfaces in $R^{3}$. Funct. Anal. Appl. 32, 4962 (1998)

69. Taimanov, I.: Two-dimensional Dirac operator and the theory of surfaces. Uspekhi Mat. Nauk 61(367), 85164 (2006)

70. Terng, C., Uhlenbeck, K.: Bäcklund transformations and loop group actions. Commun. Pure Appl. Math. LIII, 1-75 (2000)

71. Uhlenbeck, K.: Harmonic maps into Lie groups (classical solutions of the chiral model). J. Differ. Geom. 30, 1-50 (1989)

72. Uhlenbeck, K.: On the connection between harmonic maps and the self-dual Yang-Mills and the sineGordon equations. J. Geom. Phys. 8, 283-316 (1992) 
73. Weierstrass, K.: Über die Flächen, deren mittlere Krümmung überall gleich null ist, pp. 612-625. Ber. Akad. Wiss, Berlin (1866)

Publisher's Note Springer Nature remains neutral with regard to jurisdictional claims in published maps and institutional affiliations. 